\title{
The MacMahon R-matrix
}

\section{Hidetoshi Awata, ${ }^{a}$ Hiroaki Kanno, ${ }^{a, b}$ Andrei Mironov, ${ }^{c, d, e}$ Alexei Morozov, ${ }^{d, e}$ Kazuma Suetake ${ }^{a}$ and Yegor Zenkevich ${ }^{d, f, g}$}

${ }^{a}$ Graduate School of Mathematics, Nagoya University,

Nagoya 464-8602, Japan

${ }^{b}$ KMI, Nagoya University,

Nagoya 464-8602, Japan

${ }^{c}$ Theory Department, Lebedev Physics Institute,

Leninsky pr., 53, Moscow 119991, Russia

${ }^{d}$ ITEP, Bol. Cheremushkinskaya, 25, Moscow 117218, Russia

${ }^{e}$ Institute for Information Transmission Problems,

Bol. Karetny, 19 (1), Moscow 127994, Russia

${ }^{f}$ Dipartimento di Fisica, Università di Milano-Bicocca,

Piazza della Scienza 3, Milano I-20126, Italy

${ }^{g}$ INFN, sezione di Milano-Bicocca,

Milano I-20126, Italy

E-mail: awata@math.nagoya-u.ac.jp, kanno@math.nagoya-u.ac.jp, mironov@lpi.ru, morozov@itep.ru, m14020z@math.nagoya-u.ac.jp, yegor.zenkevich@gmail.com

Abstract: We introduce an $R$-matrix acting on the tensor product of MacMahon representations of Ding-Iohara-Miki (DIM) algebra $U_{q, t}\left(\widehat{\hat{\mathfrak{g l}}}_{1}\right)$. This $R$-matrix acts on pairs of $3 d$ Young diagrams and retains the nice symmetry of the DIM algebra under the permutation of three deformation parameters $q, t^{-1}$ and $\frac{t}{q}$. We construct the intertwining operator for a tensor product of the horizontal Fock representation and the vertical MacMahon representation and show that the intertwiners are permuted using the MacMahon $R$-matrix.

KeYwords: Conformal and W Symmetry, Quantum Groups, Topological Strings

ArXiv ePrint: 1810.07676 


\section{Contents}

1 Introduction 1

$1.1 q W_{1+\infty}$ algebra and the vector representation 3

2 Representations of DIM algebra 5

2.1 Definition of the quantum toroidal algebra $U_{\mathfrak{q}, \mathfrak{d}}\left(\widehat{\hat{\mathfrak{g l}}}_{1}\right)$

2.2 Vertical representations $\quad 7$

2.2.1 Vector representation $\quad 8$

2.2.2 Fock representation 8

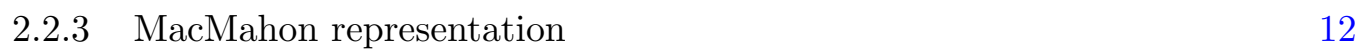

$\begin{array}{lll}2.3 & \text { Horizontal vertex operator representation } & 14\end{array}$

3 DIM $R$-matrix for MacMahon representations 16

4 Intertwining operator for MacMahon representation $\quad 17$

$\begin{array}{lll}4.1 \text { Vector intertwiner } & 18\end{array}$

$\begin{array}{lll}\text { 4.1.1 Definition of the vector intertwiner } & 18\end{array}$

$\begin{array}{ll}\text { 4.1.2 Construction of the vector intertwiner } & 19\end{array}$

$\begin{array}{lll}4.2 & \text { Fock intertwiner } & 20\end{array}$

4.2.1 Definition of the Fock intertwiner 20

4.2.2 Construction of the Fock intertwiner 21

$\begin{array}{lll}4.3 & \text { MacMahon intertwiner } & 24\end{array}$

4.3.1 Definition of the MacMahon intertwiner 24

4.3.2 Construction of the MacMahon intertwiner 24

5 MacMahon $R$-matrix from the commutation of intertwiners $\quad 27$

6 Conclusions 27

$\begin{array}{lr}\text { A Conventions and useful functions } & 29\end{array}$

B Properties of $\theta$-function $\quad 30$

C Zero modes of the Fock intertwiner 30

\section{Introduction}

Ding-Iohara-Miki (DIM) algebra [1, 2], also denoted by $U_{\mathfrak{q}, \mathfrak{d}}\left(\widehat{\widehat{\mathfrak{g l}}}_{1}\right)$, or $U_{q, t}\left(\widehat{\widehat{\mathfrak{g l}}}_{1}\right)$ is a $t$ deformation of the algebra of volume-preserving diffeomorphisms of the quantum torus $[3$, 4], which can also be described as $q W_{1+\infty}$-algebra, or a deformation of the double loop algebra of $\mathfrak{g l}_{1}=\mathbb{C}^{\times}[5]$. The DIM algebra is an extremely elegant object. It has three deformation parameters $q_{1}=q=\mathfrak{d q}^{-1}$ (coming from the quantization of the torus), $q_{2}=t^{-1}=\mathfrak{q}^{-1} \mathfrak{d}^{-1}$ (the $t$-deformation) and $q_{3}=\frac{t}{q}=\frac{1}{q_{1} q_{2}}=\mathfrak{q}^{2}$, which are treated on 
equal footing. In fact, the algebra is invariant with respect to the permutations of $q_{i}$. It also enjoys an $\mathrm{SL}(2, \mathbb{Z})$ symmetry, ${ }^{1}$ corresponding to the mapping class group of the quantum torus. There are two central charges in the algebra, which form a two-dimensional representation of the $\mathrm{SL}(2, \mathbb{Z})$ automorphism group.

A natural ${ }^{2}$ representation of the DIM algebra seems to be the MacMahon module $\mathcal{M}(K ; v)$, i.e. a linear space spanned by plane partitions, or $3 d$ Young diagrams. Here is an example of a $3 d$ Young diagram:

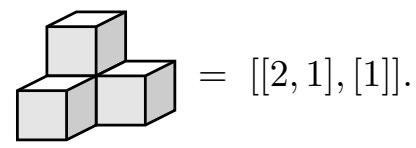

We denote the $3 d$ diagrams as sequences of ordinary Young diagrams lying at the successive horizontal layers, as seen on the r.h.s. of eq. (1.1). Metaphorically, one can think of an additional dimension in the diagrams as related to an additional loop in the definition of the algebra (compared, e.g., to the affine or Virasoro algebras). The MacMahon representation has the central charge vector $(1, K)$ (with $K$ arbitrary). Permutations of the three deformation parameters $q_{i}$ act on this representation by permutations of the axes of the $3 d$ Young diagram, i.e. by $3 d$ "transpositions".

There are other representations of the DIM algebra, which are in fact reductions of the MacMahon one. If one puts the central charge $K$ to a particular quantized value $K=q_{i}$, then the MacMahon representation becomes reducible. After factoring out the invariant subspace, one gets the space of ordinary Young diagrams. The resulting representation is called the Fock (or free field) representation $\mathcal{F}(v)$. More generally, when $K=q_{1}^{a} q_{2}^{b} q_{3}^{c}, a, b, c \in \mathbb{Z}$, the MacMahon representation reduces to a representation spanned by plane partitions with a "pit" [10,11]. Further reduction is provided by the vector representation, in which the central charges are trivial and the vectors can be thought of as columns of boxes, i.e. $1 d$ Young diagrams.

The DIM algebra is a (quasi triangular) Hopf algebra and has a universal $R$-matrix. In $[12,13]$, we have explicitly constructed the $R$-matrix acting on the product of Fock representations and elucidated its role in refined topological strings and the Nekrasov partition function. ${ }^{3}$ In the present work, we follow the same program and construct the DIM $R$-matrix for the MacMahon representations.

For the Fock spaces, there is a well-known construction [15] of intertwining operators, which turn out to be the refined topological vertices $C_{\lambda \mu \nu}(q, t)$ of topological string theory [16-23]. The labels $\lambda, \mu$ and $\nu$ in the vertex are $2 d$ Young diagrams denoting the vectors in the three Fock spaces being intertwined. Here we are going to generalize the intertwiner construction so that one of the labels in the vertex is the $3 d$ Young diagram. This indicates the existence of a generalization of refined topological string theory, in which the $3 d$ Young diagrams live on the edges along the preferred direction of the toric diagram.

\footnotetext{
${ }^{1}$ For possible extensions to $\mathrm{SL}(3, \mathbb{Z})$ symmetry see [6-9].

${ }^{2}$ Though, probably not the most general: in the MacMahon representations one of the central charges is fixed to be unit (we use the multiplicative convention for levels), while generally the both central charges can be chosen arbitrarily.

${ }^{3}$ Similar $R$-matrix was considered from the point of view of geometric representation theory in [14].
} 
The parameters $q$ and $t$ are perhaps most familiar from the theory of Macdonald polynomials [24]. The basis of Macdonald polynomials in fact plays a distinguished role in the Fock representation of DIM algebra. The $R$-matrix is then conveniently written as a set of matrix elements in the Macdonald basis. Our construction for the $R$-matrix in the MacMahon representations is similar, but the role of Macdonald polynomials is played by triple Macdonald polynomials [25].

More concretely, in this note, we try to construct the intertwining operator for the MacMahon representation as the vertical representation. In [15], the authors chose the Fock representation and constructed the intertwiner that reproduced the refined topological vertex. We want to replace the Fock representation with the MacMahon representation. One of the features of the MacMahon representation is that it has a continuous value $K$ for the second level $\gamma_{2}$ (see section 2.1 for the notation). Consequently, we also need vertex operator representations that allow $\gamma_{2}$ to be continuous. At the best of our knowledge, such a vertex operator representation is not known at the moment, though a continuous shift of $\gamma_{2}$ may be achieved by some judicious choice of the zero mode sector. To overcome this problem, we make use of the idea of constructing the MacMahon representation from an infinite tensor product of Fock representations [11]. A similar idea is employed for constructing the Fock representation from the vector representation [26]. Therefore, in order to support the validity of our approach, we first start with the intertwiner for the vector representation and construct the Fock intertwiner in a way parallel to [26] where the Fock representation was constructed from a certain infinite tensor product of vector representations. It turns out that, with this method, we can obtain the same Fock intertwiner as [15] did and, as a by-product, also an additional insight into relevant horizontal representations. Thus, we believe we can lift the construction in $[11,26]$ to the level of intertwiners.

In the remaining part of the introduction, as a warm-up, we offer a construction of the intertwiner and of the $R$-matrix for the vector representations in order to prepare for technicalities of the main text. The remaining part of the paper is organized as follows. We introduce the definition of the DIM algebra in section 2.1 and present the known representations of the algebra in sections 2.2-2.3. In particular, we review the idea of taking an infinite tensor product following [26] and [11] in section 2.2. The crucial point here is how to regularize the infinite products. In section 3 , based on the formula of the diagonal part of the universal $R$-matrix [27], we compute the $R$-matrix for the MacMahon representations. We then construct the intertwining operator for the MacMahon representations in a way parallel to $[11,26]$ in section 4 . In section 5 , we confirm that the commutator of such intertwiners indeed gives the same MacMahon $R$-matrix computed in section 3 . We present our conclusions in section 6 .

\section{1 $q W_{1+\infty}$ algebra and the vector representation}

In this part of the introduction, we deal with a "toy model" of the representations and intertwiners, which we are going to study in the main part of the paper. 
The toy algebra. Instead of the DIM algebra, we take its $t=1$ limit: $q W_{1+\infty}$ algebra, which is a Lie algebra spanned by the generators $W_{m, n}$ satisfying the relations

$$
\left[W_{m_{1}, n_{1}}, W_{m_{2}, n_{2}}\right]=\left(q^{n_{1} m_{2}}-q^{n_{2} m_{1}}\right) W_{m_{1}+m_{2}, n_{1}+n_{1}}+\left(c_{1} n_{1}+c_{2} m_{1}\right) q^{\frac{n_{1} m_{1}}{2}} \delta_{m_{1}+m_{2}, 0} \delta_{n_{1}+n_{2}, 0},
$$

where $c_{1,2}$ are the central charges. There are two grading operators $d_{1}$ and $d_{2}$ counting $m$ and $n$ in $W_{m, n}$. The relations are manifestly $\mathrm{SL}(2, \mathbb{Z})$-invariant, if we assume that the labels $(m, n)$ and the central charges $\left(c_{1}, c_{2}\right)$ transform as $2 d$ vectors under $\mathrm{SL}(2, \mathbb{Z})$ transformations.

The toy representation. We further simplify our toy model by putting $c_{1,2}=0$. Then the algebra (1.2) can be represented by difference operators acting on functions of one variable $z$ :

$$
\rho\left(W_{m, n}\right)=z^{m} q^{n z \partial_{z}} .
$$

We call representation $\rho$ the vector representation. This is nothing but the evaluation representation of the algebra (1.2). As usual, there are two viewpoints on the evaluation representation. In the first convention, the representation is seen as a finite-dimensional (in our case one-dimensional) space of functions $f(z)$, while, within the second approach, the representation is an infinite-dimensional space of vectors, in which the parameter $z$ is not a variable, but is fixed.

In the case under discussion, one can also use the equivalence of two conventions and say that the states in the vector representation, instead of being functions of $z$, are now labelled by an integer and a complex parameter, $|z, i\rangle$, so that

$$
\rho\left(W_{m, n}\right)|z, \ell\rangle=z^{m}|z, \ell+n\rangle .
$$

The parameter $z$ is fixed in this definition and is called the spectral parameter of the vector representation. Evidently, this is just a rewriting of eq. (1.3) with $|z, \ell\rangle$ corresponding to $f\left(z q^{\ell}\right)$.

The toy intertwiner. The toy algebra (1.2) is a Hopf algebra with the standard Liealgebraic coproduct

$$
\Delta\left(W_{m, n}\right)=W_{m, n} \otimes 1+1 \otimes W_{m, n} .
$$

One can then build tensor products of representations. In particular, one can take the tensor product of the Fock representation of (1.2), which has $c_{1}=\log _{q_{3}} K_{1}=1, c_{2}=$ $\log _{q_{3}} K_{2}=0$, and the vector representation.

There is an intertwining operator $\Psi$, which acts from this tensor product into a single Fock representation. It is just the standard ( $q$-deformed) vertex operator

$$
\Psi|z, \ell\rangle \otimes \cdots=\exp \left(\sum_{n \geq 1} \frac{1}{n} z^{n} q^{\ell n} a_{-n}\right) \exp \left(-\sum_{n \geq 1} \frac{1}{n} z^{-n} q^{-\ell n} a_{n}\right) .
$$

The intertwiners commute up to contact terms, which is the reflection of the fact that the coproduct (1.5) is symmetric, and thus the $R$-matrix of the algebra (1.2) is trivial. 
A small generalization and the toy $\boldsymbol{R}$-matrix. Leaving the rigorous derivation for section 4 , we give here the vector intertwining operator for $t \neq q$ (see also [28]):

$$
\Psi(|z, \ell\rangle \otimes \cdot)=\exp \left(\sum_{n \geq 1} \frac{1}{n} \frac{1-t^{n}}{1-q^{n}} t^{-n} z^{n} q^{\ell n} a_{-n}\right) \exp \left(-\sum_{n \geq 1} \frac{1}{n} q^{n} z^{-n} q^{-\ell n} a_{n}\right) .
$$

The commutation of intertwiners (1.7) yields a nontrivial (though diagonal) $R$-matrix:

$$
\Psi(|z, k\rangle \otimes \Psi(|w, \ell\rangle \otimes \cdot))=R_{k \ell}\left(\frac{z}{w}\right) \Psi(|w, \ell\rangle \otimes \Psi(|z, k\rangle \otimes \cdot)),
$$

where

$$
R_{k \ell}\left(\frac{z}{w}\right)=\frac{\left(\frac{z}{w} q^{k-\ell} t ; q\right)_{\infty}\left(\frac{z}{w} q^{k-\ell} \frac{q}{t} ; q\right)_{\infty}}{\left(\frac{z}{w} q^{k-\ell} ; q\right)_{\infty}\left(\frac{z}{w} q^{k-\ell} q ; q\right)_{\infty}}
$$

This $R$-matrix, though may seem simple, actually encodes in itself the information about all the representations of the DIM algebra. This is because all the representations can be built from the vector representation by taking appropriate tensor products so that the intertwiners also become products of the basic intertwiner (1.7). This will be precisely our strategy in the main part of the paper.

\section{Representations of DIM algebra}

\subsection{Definition of the quantum toroidal algebra $\left.U_{\mathfrak{q}, \mathfrak{d}}(\widehat{\widehat{\mathfrak{g l}}})_{1}\right)$}

The quantum toroidal algebra $U_{\mathfrak{q}, \mathfrak{d}}\left(\widehat{\hat{\mathfrak{g l}}}_{1}\right)$ has two deformation parameters $\mathfrak{q}, \mathfrak{d}$. We introduce the structure function

$$
g(z, w)=\left(z-q_{1} w\right)\left(z-q_{2} w\right)\left(z-q_{3} w\right)
$$

where our convention is

$$
q_{1}=\mathfrak{d} \mathfrak{q}^{-1}, \quad q_{2}=\mathfrak{d}^{-1} \mathfrak{q}^{-1}, \quad q_{3}=\mathfrak{q}^{2}, \quad q_{1} q_{2} q_{3}=1 .
$$

In this paper, we assume that $q_{i}$ are generic, that is, $q_{1}^{a} q_{2}^{b} q_{3}^{c}=1$ for integer $a, b, c$ if and only if $a=b=c$. We also use

$$
\begin{aligned}
G(z) & =-\frac{g(z, 1)}{g(1, z)} \\
G(w / z) & =\frac{\left(q_{1}-w / z\right)\left(q_{2}-w / z\right)\left(q_{3}-w / z\right)}{\left(1-q_{1} w / z\right)\left(1-q_{2} w / z\right)\left(1-q_{3} w / z\right)}=-\frac{g(w, z)}{g(z, w)}=G(z / w)^{-1} .
\end{aligned}
$$

The generators of $U_{\mathfrak{q}, \mathfrak{d}}\left(\widehat{\widehat{\mathfrak{g}}}_{1}\right)$ are $E_{k}, F_{k}, K^{ \pm 1}, H_{r}(k \in \mathbb{Z}, r \in \mathbb{Z} \backslash\{0\})$ and the central element $C$. It is convenient to introduce the generating functions (currents)

$$
E(z)=\sum_{k \in \mathbb{Z}} E_{k} z^{-k}, \quad F(z)=\sum_{k \in \mathbb{Z}} F_{k} z^{-k}, \quad K^{ \pm}(z)=K^{ \pm 1} \exp \left( \pm\left(\mathfrak{q}-\mathfrak{q}^{-1}\right) \sum_{r=1}^{\infty} H_{ \pm r} z^{\mp r}\right) .
$$


Note that $K^{ \pm}(z)$ is expanded in negative (positive) powers of $z$. With these currents, $U_{\mathfrak{q}, \mathfrak{d}}\left(\widehat{\mathfrak{g l}}_{1}\right)$ is defined by the following relations: ${ }^{4}$

$$
\begin{aligned}
K^{ \pm}(z) K^{ \pm}(w) & =K^{ \pm}(w) K^{ \pm}(z), \\
\frac{g\left(C^{-1} z, w\right)}{g(C z, w)} K^{-}(z) K^{+}(w) & =\frac{g\left(w, C^{-1} z\right)}{g(w, C z)} K^{+}(w) K^{-}(z), \\
g(z, w) K^{ \pm}\left(C^{(1 \mp 1) / 2} z\right) E(w)+g(w, z) E(w) K^{ \pm}\left(C^{(1 \mp 1) / 2} z\right) & =0, \\
g(w, z) K^{ \pm}\left(C^{(1 \pm 1) / 2} z\right) F(w)+g(z, w) F(w) K^{ \pm}\left(C^{(1 \pm 1) / 2} z\right) & =0, \\
{[E(z), F(w)] } & =\tilde{g}\left(\delta\left(C \frac{w}{z}\right) K^{+}(z)-\delta\left(C \frac{z}{w}\right) K^{-}(w)\right), \\
g(z, w) E(z) E(w)+g(w, z) E(w) E(z) & =0, \\
g(w, z) F(z) F(w)+g(z, w) F(w) F(z) & =0 .
\end{aligned}
$$

The coefficient $\tilde{g}$ in (2.10) affects only the relative normalization of currents $E(z), F(z)$ and $K^{ \pm}(z)$. In this paper, we choose

$$
\tilde{g}=\frac{\left(1-q_{1}\right)\left(1-q_{2}\right)}{1-q_{3}^{-1}} .
$$

There are essentially ${ }^{5}$ two central elements $C$ and $K^{-}=\left(K^{+}\right)^{-1}$, and we define that the representation has level $\left(\gamma_{1}, \gamma_{2}\right)$ if $C=\gamma_{1}$ and $K^{-}=\gamma_{2}$. We call representations with $C=1$ and $C=\mathfrak{q}$ vertical and horizontal representation, respectively. ${ }^{6}$ The commutation relation of modes $H_{r}$ are read from (2.7),

$$
\begin{aligned}
{\left[H_{r}, H_{s}\right] } & =\delta_{r+s, 0} \frac{[r]}{r}\left(\mathfrak{q}^{r}+\mathfrak{q}^{-r}-\mathfrak{d}^{r}-\mathfrak{d}^{-r}\right) \frac{C^{r}-C^{-r}}{\mathfrak{q}-\mathfrak{q}^{-1}} \\
& =\delta_{r+s, 0} \frac{[r]}{r} \mathfrak{q}^{r}\left(1-q_{1}^{r}\right)\left(1-q_{2}^{r}\right) \frac{C^{r}-C^{-r}}{\mathfrak{q}-\mathfrak{q}^{-1}}
\end{aligned}
$$

where we define $\mathfrak{q}$-integer by

$$
[r]:=\frac{\mathfrak{q}^{r}-\mathfrak{q}^{-r}}{\mathfrak{q}-\mathfrak{q}^{-1}} .
$$

Note that in the vertical representations $H_{r}$ are mutually commuting.

Actually the vertex operator representation (see section 2.3), which is the only horizontal representation to be considered in this paper, satisfies stronger relations:

$$
\begin{aligned}
& K^{ \pm}(z) K^{ \pm}(w)=K^{ \pm}(w) K^{ \pm}(z), \\
& K^{+}(z) K^{-}(w)=G\left(C^{-1} w / z\right) G(C w / z)^{-1} K^{-}(w) K^{+}(z),
\end{aligned}
$$

\footnotetext{
${ }^{4}$ They also satisfy the Serre relations, which we do not use in this paper, [2, 29].

${ }^{5}$ We impose $K^{-}=\left(K^{+}\right)^{-1}$.

${ }^{6}$ They correspond to the two choices of the Borel subalgebra, "vertical" and "horizontal" are related by the spectral duality automorphism $\mathcal{S}$ [2,30-38] (a proof is given in Lemma A.5 of [27]), which is one of the generators of the $\operatorname{SL}(2, \mathbb{Z})$ automorphism group of the DIM algebra.
} 


$$
\begin{aligned}
K^{+}(z) E(w) & =G(w / z) E(w) K^{+}(z), \\
E(z) K^{-}(C w) & =G(w / z) K^{-}(C w) E(z), \\
K^{+}(C z) F(w) & =G(w / z)^{-1} F(w) K^{+}(C z), \\
F(z) K^{-}(w) & =G(w / z)^{-1} K^{-}(w) F(z), \\
{[E(z), F(w)] } & =\tilde{g}\left(\delta\left(C \frac{w}{z}\right) K^{+}(z)-\delta\left(C \frac{z}{w}\right) K^{-}(w)\right), \\
g(z, w) E(z) E(w)+g(w, z) E(w) E(z) & =0 \\
g(w, z) F(z) F(w)+g(z, w) F(w) F(z) & =0 .
\end{aligned}
$$

To define the tensor product of two representations, we need a coproduct of $U_{\mathfrak{q}, \mathfrak{o}}\left(\widehat{\hat{\mathfrak{g l}}} \mathfrak{l}_{1}\right)$. We use the following coproduct in this paper:

$$
\begin{aligned}
\Delta(E(z)) & =E(z) \otimes 1+K^{-}\left(C_{1} z\right) \otimes E\left(C_{1} z\right), \\
\Delta(F(z)) & =F\left(C_{2} z\right) \otimes K^{+}\left(C_{2} z\right)+1 \otimes F(z), \\
\Delta\left(K^{+}(z)\right) & =K^{+}(z) \otimes K^{+}\left(C_{1}^{-1} z\right), \\
\Delta\left(K^{-}(z)\right) & =K^{-}\left(C_{2}^{-1} z\right) \otimes K^{-}(z), \\
\Delta(C) & =C \otimes C,
\end{aligned}
$$

where $C_{1}=C \otimes 1$ and $C_{2}=1 \otimes C$.

We also have two grading operators $d_{1}$ and $d_{2}$ such that

$$
\begin{aligned}
& {\left[d_{1}, E(z)\right]=-E(z), \quad\left[d_{1}, F(z)\right]=F(z), \quad\left[d_{1}, H(z)\right]=0,} \\
& {\left[d_{2}, E(z)\right]=z \partial_{z} E(z), \quad\left[d_{2}, F(z)\right]=z \partial_{z} F(z), \quad\left[d_{2}, H(z)\right]=z \partial_{z} H(z) .}
\end{aligned}
$$

\section{$2.2 \quad$ Vertical representations}

Since $C=1$ for the vertical representations, the modes $H_{r}(r \in \mathbb{Z} \backslash\{0\})$ commute with each other due to the relation (2.7). Then we can find a basis which simultaneously diagonalizes $H_{r}$. In $[11,26]$, three kinds of vertical representations: the vector, Fock and MacMahon representations have been defined. In these representations, the basis on which $H_{r}$ acts diagonally is roughly speaking labeled by $1 \mathrm{D}, 2 \mathrm{D}$ and 3D Young diagrams, respectively. Accordingly, we can define the Fock representation as an irreducible subrepresentation of an appropriate infinite tensor product of the vector representations [26]. In a similar manner, we can construct the MacMahon representation from the Fock representations [11]. The second level of the vector representation is $\gamma_{2}=1$. However, the regularization procedure required in the above procedure makes $\gamma_{2}$ non-trivial. Consequently, the Fock representation has a quantized level $\gamma_{2}=\mathfrak{q}$. Moreover, quite interestingly the MacMahon representation allows continuous level $\gamma_{2}=K$. We can find a natural regularization for the Fock representation, while that for the MacMahon representation somehow looks ambiguous and leads to an arbitrary value $K$, which we can interpret formally as a limit of $\mathfrak{q}^{N}(N \rightarrow \infty)$. In summary, the explicit actions of the vector, Fock and MacMahon representations are given by $(2.32)-(2.35),(2.57)-(2.60)$ and (2.75)-(2.77), respectively. 


\subsubsection{Vector representation}

The vector representation $V(v)$ consists of the basis $[v]_{i}$ labeled by $i \in \mathbb{Z}$ and depends on the spectral parameter $v$. The action of the algebra is defined as

$$
\begin{aligned}
K^{+}(z)[v]_{i} & =\tilde{\psi}\left(q_{1}^{i} v / z\right)[v]_{i}, \\
K^{-}(z)[v]_{i} & =\tilde{\psi}\left(q_{1}^{-i-1} z / v\right)[v]_{i}, \\
E(z)[v]_{i} & =\mathcal{E} \delta\left(q_{1}^{i+1} v / z\right)[v]_{i+1}, \\
F(z)[v]_{i+1} & =\mathcal{F} \delta\left(q_{1}^{i+1} v / z\right)[v]_{i},
\end{aligned}
$$

where the generating function of eigenvalues of $K^{ \pm}(z)$ is expressed by

$$
\tilde{\psi}(z)=\frac{\left(1-q_{2}^{-1} z\right)\left(1-q_{3}^{-1} z\right)}{(1-z)\left(1-q_{1} z\right)}=\tilde{\psi}\left(q_{1}^{-1} / z\right) .
$$

The multiplication factors $\mathcal{E}$ and $\mathcal{F}$ are determined from the choice of $\tilde{g}$ :

$$
\mathcal{E} \cdot \mathcal{F}=\tilde{g} \frac{\left(1-q_{2}^{-1}\right)\left(1-q_{3}^{-1}\right)}{1-q_{1}}=\left(1-q_{2}\right)\left(1-q_{2}^{-1}\right),
$$

and, in this paper, we choose

$$
\mathcal{E}=1-q_{2}, \quad \mathcal{F}=1-q_{2}^{-1}
$$

Since $\tilde{\psi}(0)=1$, the vector representation has the trivial level $(1,1)$. It is also convenient to introduce a more fundamental function $\psi(z)$ such that

$$
\psi(z)=\mathfrak{q} \frac{1-q_{3}^{-1} z}{1-z}=\psi\left(q_{3} / z\right)^{-1}, \quad \tilde{\psi}(z)=\psi(z) \psi\left(q_{2}^{-1} z\right)^{-1} .
$$

\subsubsection{Fock representation}

At first following [26], we outline the idea to obtain the Fock representation $(\rho, \mathcal{F}(v))$. One can construct a natural tensor product of the vector representations by making use of the coproduct of $U_{\mathfrak{q}, \mathfrak{d}}\left(\widehat{\widehat{g l}}_{1}\right)$. We want to define an infinite tensor product of the vector representations and find an irreducible subrepresentation, whose basis is composed of partitions, that is, the Fock representation. Let us consider the following tensor product with $q_{2}$-shifted spectral parameters:

$$
\left.\bigotimes_{i=1}^{N} V\left(q_{2}^{i-1} v\right) \ni \mid \lambda\right)=\bigotimes_{i=1}^{N}\left[q_{2}^{i-1} v\right]_{\lambda_{i}-1}, \quad \lambda=\left(\lambda_{1}, \ldots, \lambda_{N}\right) \in \mathbb{Z}^{N}
$$

Since $C=1$ for the vector representations, the coproduct (2.27) and (2.28) give

$$
\Delta^{N-1}\left(K^{ \pm}(z)\right)=\overbrace{K^{ \pm}(z) \otimes \cdots \otimes K^{ \pm}(z)}^{N} .
$$


Similarly, the coproduct (2.25) and (2.26) implies

$$
\begin{aligned}
& \Delta^{N-1}(E(z))=\sum_{k=1}^{N} \overbrace{K^{-}(z) \otimes \cdots K^{-}(z)}^{k-1} \otimes E(z) \otimes \overbrace{1 \otimes \cdots \otimes 1}^{N-k}, \\
& \Delta^{N-1}(F(z))=\sum_{k=1}^{N} \overbrace{1 \otimes \cdots \otimes 1}^{k-1} \otimes F(z) \otimes \overbrace{K^{+}(z) \otimes \cdots \otimes K^{+}(z)}^{N-k} .
\end{aligned}
$$

The tensor product representation is defined by $\rho_{N}(X(z)):=\rho_{u_{1}}^{\mathrm{v}} \otimes \cdots \otimes \rho_{u_{N}}^{\mathrm{v}}\left(\Delta^{N-1}(X(z))\right)$, where $\rho_{u_{i}}^{\mathrm{v}}$ denotes the vector representation with the spectral parameter $u_{i}=q_{2}^{i-1} v$.

One can naturally view $\lambda \in \mathbb{Z}^{N}$ as $\lambda \in \mathbb{Z}^{N+1}$ with $\lambda_{N+1}=0$. However $\left(\rho_{N}, \bigotimes_{i=1}^{N} V\left(q_{2}^{i-1} v\right)\right)$ does not form an inductive system because actions of $\rho_{N}$ and $\rho_{N+1}$ on $\mid \lambda)\left(\lambda \in \mathbb{Z}^{N}\right)$ are different. For this reason, one cannot take a limit $N \rightarrow \infty$ naively. In order to settle this problem, we modify the action of $\left(\rho_{N}, \bigotimes_{i=1}^{N} V\left(q_{2}^{i-1} v\right)\right)$ to some $\left(\bar{\rho}_{N}, \bigotimes_{i=1}^{N} V\left(q_{2}^{i-1} v\right)\right)$ so as to form an inductive system of $U_{\mathfrak{q}, \mathfrak{d}}\left(\widehat{\hat{\mathfrak{g}}}_{1}\right)$-modules. Then one can take the inductive limit $\rho=\lim _{N \rightarrow \infty} \bar{\rho}_{N}$ to find the Fock representation $(\rho, \mathcal{F}(v))$ as an irreducible subrepresentation of $\rho$ :

$$
\left.\bigotimes_{i=1}^{\infty} V\left(q_{2}^{i-1} v\right) \supset \mathcal{F}(v) \ni \mid \lambda\right)=\bigotimes_{i=1}^{\infty}\left[q_{2}^{i-1} v\right]_{\lambda_{i}-1},
$$

where $\lambda=\left(\lambda_{1}, \ldots, \lambda_{\ell(\lambda)}\right) \in \mathcal{P}$ : set of partitions and $\lambda_{n}=0(n>\ell(\lambda))$.

In the following, we describe the idea of modification in detail. Actually, we have to modify only $\rho_{N}\left(K^{ \pm}(z)\right)$ and $\rho_{N}(F(z))$ keeping $\rho_{N}(E(z))$ intact. Let us modify the action of $\rho_{N}$ to $\bar{\rho}_{N}$ so as to obtain the condition, for $X=K^{ \pm}, E$ or $F$,

$$
\left.\bar{\rho}_{N}(X(z))=\bar{\rho}_{N+M}(X(z)) \text { on } \mid \lambda\right), \lambda \in \mathbb{Z}^{N-1}, \quad \forall M \in \mathbb{N}
$$

Then we can define the action of $\rho(X(z))$ on $\mid \lambda)\left(\lambda \in \mathbb{Z}^{N-1}\right)$ as $\bar{\rho}_{N}(X(z))$. Therefore, we should search for a modified action which satisfies the condition (2.45). At first, let us see that the action $\bar{\rho}_{N}(E(z))$ can be the same as $\rho_{N}(E(z))$. This action satisfies the condition (2.45) due to the vanishing property,

$$
\begin{aligned}
& \left(K^{-}(z) \otimes E(z)\right)\left(\left[q_{2}^{N-1} v\right]_{-1} \otimes\left[q_{2}^{N} v\right]_{-1}\right) \\
& \quad=\tilde{\psi}\left(q_{2}^{-N+1} z / v\right) \delta\left(q_{2}^{N} v / z\right)\left[q_{2}^{N-1} v\right]_{-1} \otimes\left[q_{2}^{N} v\right]_{-1}=0 .
\end{aligned}
$$

Further, let us focus on the action of $K^{ \pm}(z)$. This time the actions of $\rho_{N}$ and $\rho_{N+M}$ differ. We denote $K_{N}^{ \pm}(z)=\bar{\rho}_{N}\left(K^{ \pm}(z)\right)=\rho_{N}\left(K^{ \pm}(z)\right) \times \beta_{N}^{ \pm}=\Delta^{N-1}\left(K^{ \pm}(z)\right) \times \beta_{N}^{ \pm}$, where $\beta_{N}^{ \pm}=\beta_{N}^{ \pm}\left((v / z)^{ \pm 1}\right)$ is a modification factor that satisfies $\beta_{N}^{+}(v / z)=\beta_{N}^{-}(z / v)$ as a rational function. Since

$$
K^{+}(z)\left[q_{2}^{N} v\right]_{-1}=\tilde{\psi}\left(q_{1}^{-1} q_{2}^{N} v / z\right)\left[q_{2}^{N} v\right]_{-1},
$$

one gets a recursion relation for the modification factor $\beta_{N}^{+}$

$$
1=\frac{\left(\lambda\left|K_{N+1}^{+}(z)\right| \lambda\right)}{\left(\lambda\left|K_{N}^{+}(z)\right| \lambda\right)}=\frac{\beta_{N+1}^{+}}{\beta_{N}^{+}} \tilde{\psi}\left(q_{1}^{-1} q_{2}^{N} v / z\right)
$$


This is equivalent to

$$
\begin{aligned}
\beta_{N}^{+} & =\tilde{\psi}\left(q_{1}^{-1} q_{2}^{N-1} v / z\right)^{-1} \beta_{N-1}^{+}=\psi\left(q_{1}^{-1} q_{2}^{N-1} v / z\right)^{-1} \psi\left(q_{1}^{-1} q_{2}^{N-2} v / z\right) \beta_{N-1}^{+} \\
& =\psi\left(q_{1}^{-1} q_{2}^{N-1} v / z\right)^{-1} \psi\left(q_{1}^{-1} v / z\right) \beta_{1}^{+}
\end{aligned}
$$

which determines $\beta_{N}^{ \pm}$up to an appropriate initial condition. Naturally, the initial condition should be related to the regularization problem of the vacuum of the Fock representation. To see this, let us look at the unmodified representation $\rho_{N}$ on the "vacuum state $\left.\mid \varnothing\right)$ ", $\varnothing=(0, \ldots, 0)$,

$$
\begin{aligned}
\left(\varnothing\left|\Delta^{N-1}\left(K^{+}(z)\right)\right| \varnothing\right) & =\prod_{k=1}^{N} \tilde{\psi}\left(q_{1}^{-1} q_{2}^{k-1} v / z\right)=\prod_{k=1}^{N} \psi\left(q_{1}^{-1} q_{2}^{k-1} v / z\right) \psi\left(q_{2}^{k-1} q_{3} z / v\right)^{-1} \\
& =\frac{1-q_{2}^{N} v / z}{1-q_{2}^{N} q_{3} v / z} \frac{1-q_{3} v / z}{1-v / z} .
\end{aligned}
$$

This expression makes no sense in the limit of $N \rightarrow \infty$, but we can formally regularize it by specifying the ordering of infinite products,

$$
\psi\left(q_{3} v / z\right)^{-1} \prod_{k \geq 1}\left(\psi\left(q_{1}^{-1} q_{2}^{k-1} v / z\right) \psi\left(q_{2}^{k} q_{3} v / z\right)^{-1}\right)=\psi\left(q_{3} v / z\right)^{-1}
$$

Therefore, in the modified representation $\bar{\rho}_{N}$, one expects the vacuum expectation value to be

$$
\begin{aligned}
\left(\varnothing\left|K_{N}^{+}(z)\right| \varnothing\right) & =\prod_{k=1}^{N} \tilde{\psi}\left(q_{1}^{-1} q_{2}^{k-1} v / z\right) \beta_{N}^{+}(v / z) \\
& =\psi\left(q_{3} v / z\right)^{-1}=\mathfrak{q}^{-1} \frac{1-q_{3} v / z}{1-v / z}, \quad \forall N \in \mathbb{N} .
\end{aligned}
$$

Thus, the problematic factor $\frac{1-q_{2}^{N} v / z}{1-q_{2}^{N} q_{3} v / z}$ in $(2.51)$ has been replaced by the factor $\mathfrak{q}^{-1}$ by the regularization. Now (2.53) leads to the initial condition

$$
\beta_{1}^{+}(v / z)=\psi\left(q_{1}^{-1} v / z\right)^{-1}
$$

Hence, the above prescription for the regularization gives

$$
\beta_{N}^{+}(v / z)=\psi\left(q_{1}^{-1} q_{2}^{N-1} v / z\right)^{-1}=\psi\left(q_{2}^{-N} z / v\right)=\beta_{N}^{-}(z / v) .
$$

As concerns the action of $F(z)$, we also need some modification factor. This factor should be precisely the same as $\beta_{N}^{+}(v / z)$ for the sake of (2.10). In fact, it satisfies the condition (2.45) due to the vanishing property,

$$
\beta_{N+1}^{+} F(z)\left[q_{2}^{N} v\right]_{-1}=\psi\left(q_{1}^{-1} q_{2}^{N} v / z\right)^{-1} \delta\left(q_{1}^{-1} q_{2}^{N} v / z\right)\left[q_{2}^{N} v\right]_{-2}=0
$$


Now we can write down the action of the infinite tensor product representation $(\rho, \mathcal{F}(v))$ explicitly (see $(2.41)-(2.43))$,

$$
\begin{aligned}
\left.K^{+}(z) \mid \lambda\right) & \left.\left.=\prod_{s=1}^{\ell(\lambda)} \tilde{\psi}\left(x_{s} v / z\right) \beta_{\ell(\lambda)}^{+}(v / z) \mid \lambda\right)=\prod_{s=1}^{\ell(\lambda)} \psi\left(x_{s} v / z\right) \prod_{s=1}^{\ell(\lambda)+1} \psi\left(q_{2}^{-1} x_{s} v / z\right)^{-1} \mid \lambda\right), \\
\left.K^{-}(z) \mid \lambda\right) & \left.\left.=\prod_{s=1}^{\ell(\lambda)} \tilde{\psi}\left(q_{1}^{-1} x_{s}^{-1} z / v\right) \beta_{\ell(\lambda)}^{-}(z / v) \mid \lambda\right)=\prod_{s=1}^{\ell(\lambda)} \psi\left(q_{3} x_{s}^{-1} z / v\right)^{-1} \prod_{s=1}^{\ell(\lambda)+1} \psi\left(q_{1}^{-1} x_{s}^{-1} z / v\right) \mid \lambda\right), \\
E(z) \mid \lambda) & \left.=\left(1-q_{2}\right) \sum_{j=1}^{\ell(\lambda)+1} \prod_{s=1}^{j-1} \tilde{\psi}\left(q_{1}^{-1} x_{s} / x_{j}\right) \delta\left(q_{1} x_{j} v / z\right) \mid \lambda+1_{j}\right) \\
& \left.=\left(1-q_{2}\right) \sum_{j=1}^{\ell(\lambda)+1} \prod_{s=1}^{j-1} \psi\left(q_{1}^{-1} x_{s} / x_{j}\right) \psi\left(q_{3} x_{s} / x_{j}\right)^{-1} \delta\left(q_{1} x_{j} v / z\right) \mid \lambda+1_{j}\right), \\
F(z) \mid \lambda) & \left.=\left(1-q_{2}^{-1}\right) \sum_{j=1}^{\ell(\lambda)} \prod_{s=j+1}^{\ell(\lambda)} \tilde{\psi}\left(x_{s} / x_{j}\right) \delta\left(x_{j} v / z\right) \beta_{\ell(\lambda)}^{+}\left(1 / x_{j}\right) \mid \lambda-1_{j}\right) \\
& \left.=\left(1-q_{2}^{-1}\right) \sum_{j=1}^{\ell(\lambda)} \prod_{s=j+1}^{\ell(\lambda)} \psi\left(x_{s} / x_{j}\right) \prod_{s=j+1}^{\ell(\lambda)+1} \psi\left(q_{2}^{-1} x_{s} / x_{j}\right)^{-1} \delta\left(x_{j} v / z\right) \mid \lambda-1_{j}\right),
\end{aligned}
$$

where we have introduced the coordinates $x_{s}=x_{s, \lambda_{s}}, x_{i, j}=q_{1}^{j-1} q_{2}^{i-1}$. As already mentioned, we can find the invariant subspace $\mathcal{F}(v)$ which consists of only partitions by investigating the positions of zeros appearing in the action of the creation operator $E(z)$ and the annihilation operator $F(z)[26]$. This irreducible subrepresentation generated by the empty Young diagram $\varnothing$ is called Fock representation. The Fock representation is the highest weight representation with the empty Young diagram $\varnothing$ being the highest weight state. The generating function of eigenvalues of the vacuum is $\left(\varnothing\left|K^{+}(z)\right| \varnothing\right)=\psi\left(q_{3} v / z\right)^{-1}$. Note that, similarly to the vector representation, the finite tensor representations have trivial level $(1,1)$, however, now the Fock representation has the nontrivial level $(1, \mathfrak{q})$ due to the chosen regularization (2.53).

Thanks to the relation

$$
G\left(x_{i, j} v / z\right)=\tilde{\psi}\left(x_{i, j} v / z\right) \tilde{\psi}\left(q_{1}^{-1} x_{i, j} v / z\right)^{-1}
$$

one can rewrite the above formula in a symmetric way, for example,

$$
\left(\lambda\left|K^{+}(z)\right| \lambda\right)=\psi_{\varnothing}(v / z) \prod_{(i, j) \in \lambda} G\left(x_{i, j} v / z\right)
$$

where $\psi_{\varnothing}(v / z)$ is

$$
\psi_{\varnothing}(v / z)=\left(\varnothing\left|K^{+}(z)\right| \varnothing\right)=\psi\left(q_{3} v / z\right)^{-1}
$$




\subsubsection{MacMahon representation}

We want to define the MacMahon representation as an appropriate limit of $\left(\pi_{N}, \mathcal{M}_{N}(v)\right)$ similarly to the definition of the Fock representation [11],

$$
\left.\left.\bigotimes_{k=1}^{N} \mathcal{F}\left(q_{3}^{k-1} v\right) \supset \mathcal{M}_{N}(v) \ni \mid \Lambda\right)=\bigotimes_{k=1}^{N} \mid \Lambda^{(k)}\right)
$$

The notation of the plane partition (3d partition) $\Lambda$ is as follows

$$
\Lambda=\left(\Lambda^{(1)}, \ldots, \Lambda^{(N)}\right), \quad \Lambda^{(k)}:(2 \mathrm{~d}) \text { partition such that } \Lambda_{i}^{(k+1)} \leq \Lambda_{i}^{(k)},(\forall i, k),
$$

and we also use the notations

$$
\begin{aligned}
h(\Lambda) & =\min \{k \geq 0 \mid(1,1, k+1) \notin \Lambda\}, \\
(i, j, k) \in \Lambda & \Leftrightarrow \Lambda_{i}^{(k)} \geq j .
\end{aligned}
$$

We interpret as $\left.\mid \Lambda^{(k)}\right) \in \mathcal{F}\left(q_{3}^{k-1} v\right)$ unless otherwise mentioned.

Let us now focus on the action of $K^{ \pm}(z)$ and the regularization problem. We denote as $K_{N}^{ \pm}(z)=\bar{\pi}_{N}\left(K^{ \pm}(z)\right)=\Delta^{N-1}\left(K^{ \pm}(z)\right) \times \gamma_{N}^{ \pm}$, where $\gamma_{N}^{ \pm}=\gamma_{N}^{ \pm}\left((v / z)^{ \pm}\right)$is a modification factor which satisfies $\gamma_{N}^{+}(v / z)=\gamma_{N}^{-}(z / v)$ as a rational function. Since we have, for $\mid \varnothing) \in \mathcal{F}\left(q_{3}^{N} v\right)$,

$$
\left.\left(\varnothing\left|K^{+}(z)\right| \varnothing\right)\right|_{\mathcal{F}\left(q_{3}^{N} v\right)}=\psi\left(q_{3}^{N+1} v / z\right)^{-1},
$$

we get a recursion relation for the modification factor $\gamma_{N}^{+}$as

$$
1=\frac{\left.\left(\Lambda\left|K_{N+1}^{+}(z)\right| \Lambda\right)\right|_{\mathcal{M}_{N+1}(v)}}{\left.\left(\Lambda\left|K_{N}^{+}(z)\right| \Lambda\right)\right|_{\mathcal{M}_{N}(v)}}=\frac{\gamma_{N+1}^{+}}{\gamma_{N}^{+}} \psi\left(q_{3}^{N+1} v / z\right)^{-1},
$$

which means

$$
\gamma_{N}^{+}=\psi\left(q_{3}^{N} v / z\right) \gamma_{N-1}^{+}=\mathfrak{q}^{N-1} \frac{1-q_{3} v / z}{1-q_{3}^{N} v / z} \gamma_{1}^{+}
$$

Hence, we can determines $\gamma_{N}^{ \pm}$up to an appropriate initial condition. As concerns the initial condition, let us look at the unmodified representation $\pi_{N}$ on the vacuum state $\mid \varnothing) \in \mathcal{M}_{N}(v), \varnothing=\left(\varnothing^{(1)}, \ldots, \varnothing^{(N)}\right)$,

$$
\left.\left(\varnothing\left|\Delta^{N-1}\left(K^{+}(z)\right)\right| \varnothing\right)\right|_{\mathcal{M}_{N}(v)}=\prod_{k=1}^{N} \psi\left(q_{3}^{k} v / z\right)^{-1}=\mathfrak{q}^{-N} \prod_{k=1}^{N} \frac{1-q_{3}^{k} v / z}{1-q_{3}^{k-1} v / z}=\mathfrak{q}^{-N} \frac{1-q_{3}^{N} v / z}{1-v / z} .
$$

This expression makes no sense in the limit of $N \rightarrow \infty$, but we cannot use the same strategy as in the previous subsection due to the presence of the monomial factor $\mathfrak{q}^{-N}$. Here we have to regularize it in another way: we formally substitute $\mathfrak{q}^{N}$ by an arbitrary parameter $K^{1 / 2}$. Then, in the modified representation $\bar{\pi}_{N}$, we have

$$
\left.\left(\varnothing\left|K_{N}^{+}(z)\right| \varnothing\right)\right|_{\mathcal{M}_{N}(v)}=\prod_{k=1}^{N} \psi\left(q_{3}^{k} v / z\right)^{-1} \gamma_{N}^{+}(v / z)=K^{-1 / 2} \frac{1-K v / z}{1-v / z},
$$


which leads to the initial condition

$$
\gamma_{1}^{+}(v / z)=\frac{K^{-1 / 2}(1-K v / z)}{\mathfrak{q}^{-1}\left(1-q_{3} v / z\right)} .
$$

Hence, our regularization gives

$$
\gamma_{N}^{+}(v / z)=\frac{K^{-1 / 2}(1-K v / z)}{\mathfrak{q}^{-N}\left(1-q_{3}^{N} v / z\right)} .
$$

Now we can write down the action of the MacMahon representation $(\pi, \mathcal{M}(K ; v))$,

$$
\begin{aligned}
\left.K^{ \pm}(z) \mid \Lambda\right)= & \left.\left.\prod_{k=1}^{h(\Lambda)}\left(\Lambda^{(k)}\left|K^{ \pm}(z)\right| \Lambda^{(k)}\right)\right|_{\mathcal{F}\left(q_{3}^{k-1} v\right)} \gamma_{h(\Lambda)}^{ \pm}\left((v / z)^{ \pm 1}\right) \mid \Lambda\right) \\
= & \left.\boldsymbol{\psi}_{\varnothing}^{ \pm}\left(K^{1 / 2} ;(v / z)^{ \pm 1}\right) \prod_{(i, j, k) \in \Lambda} G\left(\left(x_{i j k} v / z\right)^{ \pm 1}\right)^{ \pm 1} \mid \Lambda\right) \\
E(z) \mid \Lambda)= & \left.\sum_{k=1}^{h(\Lambda)+1} \sum_{i=1}^{\ell\left(\Lambda^{(k)}\right)+1} \prod_{s=1}^{k-1}\left(\Lambda^{(s)}\left|K^{-}\left(q_{1} x_{i j k} v\right)\right| \Lambda^{(s)}\right)\right|_{\mathcal{F}\left(q_{3}^{s-1} v\right)} \\
& \left.\times\left.\left(\Lambda^{(k)}+1_{i}|E(z)| \Lambda^{(k)}\right)\right|_{\mathcal{F}\left(q_{3}^{k-1} v\right)} \mid \Lambda+1_{i}^{(k)}\right) \\
& \left.\quad \sum_{k=1}^{h(\Lambda)} \sum_{i=1}^{\ell\left(\Lambda^{(k)}\right)}\left(\Lambda^{(k)}-1_{i}|F(z)| \Lambda^{(k)}\right)\right|_{\mathcal{F}\left(q_{3}^{k-1} v\right)} \\
& \left.\times\left.\prod_{s=k+1}^{h(\Lambda)}\left(\Lambda^{(s)}\left|K^{+}\left(x_{i j k} v\right)\right| \Lambda^{(s)}\right)\right|_{\mathcal{F}\left(q_{3}^{s-1} v\right)} \gamma_{h(\Lambda)}^{+}\left(x_{i j k}^{-1}\right) \mid \Lambda-1_{i}^{(k)}\right)
\end{aligned}
$$

where we have introduced the coordinates $x_{i j k}=q_{1}^{j-1} q_{2}^{i-1} q_{3}^{k-1}=q_{1}^{j} q_{2}^{i} q_{3}^{k}$ and the generating function of eigenvalues of the vacuum:

$$
\boldsymbol{\psi}_{\varnothing}^{ \pm}\left(K^{1 / 2} ; u\right)=K^{\mp 1 / 2} \frac{1-K^{ \pm} u}{1-u} .
$$

We note that $j=\Lambda_{i}^{(k)}$ is understood at the r.h.s. of (2.76) and (2.77). The action of $K^{ \pm}(z)$ has a manifestly symmetric expression with respect to the permutation of $q_{1,2,3}$ but $E(z), F(z)$ do not. In [11], it was proved that the actions (2.75)-(2.77) indeed keep the subspace spanned by plane partitions. This is assured by the $q_{3}$-shift of spectral parameters among adjacent Fock representations in the tensor product. One can check that the factor $\left.\left(\Lambda^{(s)}\left|K^{-}\left(q_{1} x_{i j k} v\right)\right| \Lambda^{(s)}\right)\right|_{\mathcal{F}\left(q_{3}^{s-1} v\right)}$ in $(2.76)$ vanishes for $s=k-1$ when $\Lambda_{i}^{(k-1)}=\Lambda_{i}^{(k)}$, which means that the action of $E(z)$ cannot add the box at $\left(i, \Lambda_{i}^{(k)}+1, k\right)$. On the other hand, the factor $\left.\left(\Lambda^{(s)}\left|K^{+}\left(x_{i j k} v\right)\right| \Lambda^{(s)}\right)\right|_{\mathcal{F}\left(q_{3}^{s-1} v\right)}$ in (2.77) vanishes for $s=k+1$ when $\Lambda_{i}^{(k)}=\Lambda_{i}^{(k+1)}$, which implies that the action of $F(z)$ cannot remove the box at $\left(i, \Lambda_{i}^{(k)}, k\right)$. Thus the invariant subspace $\mathcal{M}(K ; v)$ is generated by acting $E(z)$ on the empty plane partition, which is the highest weight state, while the action of $F(z)$ keeps $\mathcal{M}(K ; v)$. In fact, there is a formula called shell formula [11,39], which expresses the r.h.s. of (2.76) and (2.77) as 
a finite sum over the concave and the convex corners of $\Lambda$. Note that $\pi_{N}$ has level $\left(1, \mathfrak{q}^{N}\right)$ as an $N$-fold Fock representation, however, now the MacMahon representation has level $\left(1, K^{1 / 2}\right)$ with a continuous parameter $K$ due to the regularization (2.72).

\subsection{Horizontal vertex operator representation}

When $C=\mathfrak{q}$, the Heisenberg part satisfies

$$
\left[H_{r}, H_{s}\right]=\delta_{r+s, 0} \frac{[r]^{2}}{r} \mathfrak{q}^{r}\left(1-q_{1}^{r}\right)\left(1-q_{2}^{r}\right),
$$

and we define the fundamental vertex operator as follows,

$$
V^{ \pm}(z)=\exp \left(\mp \sum_{r=1}^{\infty} \frac{H_{ \pm r}}{[r]} z^{\mp r}\right) .
$$

The fundamental OPE by the normal ordering is

$$
V^{+}(z) V^{-}(w)=s(w / z): V^{+}(z) V^{-}(w):,
$$

where the scattering factor $s(z)$ is

$$
s(z)=\frac{(1-\mathfrak{q} z)\left(1-\mathfrak{q}^{-1} z\right)}{(1-\mathfrak{d} z)\left(1-\mathfrak{d}^{-1} z\right)}
$$

and satisfies the following formulas as a rational function,

$$
s(z)=s\left(z^{-1}\right), \quad G(z)=s\left(\mathfrak{q}^{-1} z\right) s(\mathfrak{q} z)^{-1}=G\left(z^{-1}\right)^{-1} .
$$

We can define the vertex operator representation of level $(\mathfrak{q}, 1)$ by making use of the fundamental vertex operator as follows:

$$
\begin{gathered}
E(z) \rightarrow \eta(z)=V^{-}\left(\mathfrak{q}^{-\frac{1}{2}} z\right) V^{+}\left(\mathfrak{q}^{\frac{1}{2}} z\right), \\
F(z) \rightarrow \xi(z)=V^{-}\left(\mathfrak{q}^{\frac{1}{2}} z\right)^{-1} V^{+}\left(\mathfrak{q}^{-\frac{1}{2}} z\right)^{-1}, \\
K^{ \pm}\left(\mathfrak{q}^{1 / 2} z\right) \rightarrow \varphi^{ \pm}(z)=V^{ \pm}\left(\mathfrak{q}^{ \pm 1} z\right) V^{ \pm}\left(\mathfrak{q}^{\mp 1} z\right)^{-1},
\end{gathered}
$$

where the vacuum state $|0\rangle$ of the Fock space for the horizontal representation is defined by the annihilation condition

$$
H_{r}|0\rangle=0, \quad r>0 .
$$

The shift of the argument in $K^{ \pm}(z)$ is conventional. Furthermore, for any $\gamma_{2} \in \mathbb{C}^{\times}$, we can employ a more general level $\left(\mathfrak{q}, \gamma_{2}\right)$ representation with zero modes $\mathbf{e}(z), \mathbf{f}(z), \mathbf{k}^{ \pm}(z)$,

$$
\begin{aligned}
E(z) & \rightarrow \eta(z) \mathbf{e}(z), \\
F(z) & \rightarrow \xi(z) \mathbf{f}(z), \\
K^{ \pm}\left(\mathfrak{q}^{1 / 2} z\right) & \rightarrow \varphi^{ \pm}(z) \mathbf{k}^{ \pm}(z),
\end{aligned}
$$


where the constant part of $\mathbf{k}^{ \pm}(z)$ is fixed by the second level $\gamma_{2}$. We denote the horizontal representation with these zero modes by $\mathcal{H}=\mathcal{H}\left(\mathbf{k}^{ \pm}(z), \mathbf{e}(z), \mathbf{f}(z)\right)$. The zero modes must satisfy appropriate relations. In fact, we have

$$
\mathbf{e}(z) \mathbf{f}\left(\mathfrak{q}^{\mp 1} z\right)=\mathbf{k}^{ \pm}\left(\mathfrak{q}^{\mp 1 / 2} z\right)
$$

from (2.10), so $\mathbf{e}(z)$ can be determined from $\mathbf{f}(z)$ and $\mathbf{k}^{ \pm}(z)$ uniquely

$$
\mathbf{e}(z)=\mathbf{k}^{ \pm}\left(\mathfrak{q}^{\mp 1 / 2} z\right) / \mathbf{f}\left(\mathfrak{q}^{\mp 1} z\right)=\sqrt{\mathbf{k}^{+}\left(\mathfrak{q}^{-1 / 2} z\right) \mathbf{k}^{-}\left(\mathfrak{q}^{1 / 2} z\right) / \mathbf{f}\left(\mathfrak{q}^{-1} z\right) \mathbf{f}(\mathfrak{q} z)} .
$$

Furthermore, we make an ansatz that $\mathbf{k}^{ \pm}(z)$ is independent of $z$ :

$$
\mathbf{k}^{ \pm}(z)=\mathbf{k}^{ \pm}(0)=\gamma_{2}^{\mp 1}
$$

so that we can lift the modification factors $(2.55),(2.74)$ of vertical representations to vertex operators uniquely. Under the ansatz (2.93), there is a one parameter family of constant solutions to (2.91). However, as we will see in section 4, one cannot keep $\mathbf{e}(z)$ and $\mathbf{f}(z)$ constant for the existence of the intertwiner. For example, the level $\left(\mathfrak{q}, \mathfrak{q}^{N}\right)$ representation used in [15] is defined by

$$
\mathbf{k}^{ \pm}(z)=\mathfrak{q}^{\mp N}, \quad \mathbf{e}(z)=(\mathfrak{q} / z)^{N} u, \quad \mathbf{f}(z)=(\mathfrak{q} / z)^{-N} u^{-1}
$$

where $u$ is the spectral parameter of the representation. With the notation introduced above, we can express this horizontal representation as $\mathcal{F}_{u}^{\left(\mathfrak{q}, \mathfrak{q}^{N}\right)}=\mathcal{H}\left(\mathfrak{q}^{\mp N},(\mathfrak{q} / z)^{N} u,(\mathfrak{q} / z)^{-N} u^{-1}\right)$.

It is also useful to introduce the dual vertex operator $\tilde{V}^{ \pm}(z)$ that satisfies

$$
\begin{aligned}
& V^{+}(z) \tilde{V}^{-}(w)=(1-w / z)^{-1}: V^{+}(z) \tilde{V}^{-}(w): \\
& \tilde{V}^{+}(z) V^{-}(w)=(1-w / z): \tilde{V}^{+}(z) V^{-}(w):
\end{aligned}
$$

It is expressed explicitly as

$$
\tilde{V}^{ \pm}(z)=\exp \left(\mp \sum_{r=1}^{\infty} \Lambda_{ \pm r} z^{\mp r}\right), \quad \Lambda_{r}:=\frac{\mathfrak{q}-\mathfrak{q}^{-1}}{k_{r}} H_{r}
$$

where

$$
k_{r}=\prod_{i=1}^{3}\left(q_{i}^{r / 2}-q_{i}^{-r / 2}\right)=\prod_{i=1}^{3}\left(q_{i}^{r}-1\right)=\sum_{i=1}^{3}\left(q_{i}^{r}-q_{i}^{-r}\right)
$$

and we have

$$
\left[\Lambda_{r}, H_{s}\right]=\delta_{r+s, 0} \frac{[r]}{r} .
$$




\section{$3 \quad$ DIM $R$-matrix for MacMahon representations}

In this section, we calculate the universal DIM $R$-matrix in the basis of generalized triple Macdonald polynomials. It will be diagonal and depend on the ratio of spectral parameters $x=\frac{u}{v}$, two central charges $K_{1}, K_{2}$ and a pair of plane partitions $\Pi, \Lambda$.

We use the formula for the diagonal (on the vertical representations) part of the universal $R$-matrix from [27]:

$$
\mathcal{R}_{0}=(K \otimes 1)^{\frac{1}{2}\left(1 \otimes d_{1}\right)}(1 \otimes K)^{\frac{1}{2}\left(d_{1} \otimes 1\right)} \exp \left\{-\sum_{n \geq 1} n k_{n}\left(h_{-n} \otimes h_{n}\right)\right\},
$$

where $K$ is the second component of the central charge vector, $d_{1}$ is the first grading operator and $h_{n}$ are modes of the $K^{ \pm}(z)$ currents defined as follows:

$$
K^{ \pm}(z)=K^{\mp \frac{1}{2}} \exp \left\{\sum_{n \geq 1} k_{n} h_{ \pm n} z^{\mp n}\right\}
$$

For the vertical MacMahon representation, we have

$$
\begin{aligned}
& \left.K^{+}(z) \mid \Pi, u\right)= \\
& \left.\boldsymbol{\psi}_{\varnothing}^{+}\left(K^{1 / 2} ; \frac{u}{z}\right) \prod_{(i, j, k) \in \Pi} \frac{\left(1-q_{1}^{i-1} q_{2}^{j} q_{3}^{k} \frac{u}{z}\right)\left(1-q_{1}^{i} q_{2}^{j-1} q_{3}^{k} \frac{u}{z}\right)\left(1-q_{1}^{i} q_{2}^{j} q_{3}^{k-1} \frac{u}{z}\right)}{\left(1-q_{1}^{i+1} q_{2}^{j} q_{3}^{k} \frac{u}{z}\right)\left(1-q_{1}^{i} q_{2}^{j+1} q_{3}^{k} \frac{u}{z}\right)\left(1-q_{1}^{i} q_{2}^{j} q_{3}^{k+1} \frac{u}{z}\right)} \mid \Pi, u\right), \\
& \left.K^{-}(z) \mid \Pi, u\right)= \\
& \left.\boldsymbol{\psi}_{\varnothing}^{-}\left(K^{1 / 2} ; \frac{z}{u}\right) \prod_{(i, j, k) \in \Pi} \frac{\left(1-q_{1}^{1-i} q_{2}^{-j} q_{3}^{-k} \frac{z}{u}\right)\left(1-q_{1}^{-i} q_{2}^{1-j} q_{3}^{-k} \frac{z}{u}\right)\left(1-q_{1}^{-i} q_{2}^{-j} q_{3}^{1-k} \frac{z}{u}\right)}{\left(1-q_{1}^{-i-1} q_{2}^{-j} q_{3}^{-k} \frac{z}{u}\right)\left(1-q_{1}^{-i} q_{2}^{-j-1} q_{3}^{-k} \frac{z}{u}\right)\left(1-q_{1}^{-i} q_{2}^{-j} q_{3}^{-k-1} \frac{z}{u}\right)} \mid \Pi, u\right),
\end{aligned}
$$

and therefore

$$
\left.\left.h_{n} \mid \Pi, u\right)=\frac{u^{n}}{|n|}\left[\frac{1-K^{n}}{k_{n}}+\sum_{(i, j, k) \in \Pi} q_{1}^{n i} q_{2}^{n j} q_{3}^{n k}\right] \mid \Pi, u\right) .
$$

The $R$-matrix in two MacMahon representations is given by (we divide by the vacuum matrix element to get rid of the overall scalar factor, which can be evaluated separately)

$$
\begin{aligned}
R_{\Pi \Lambda}^{K_{1}, K_{2}}\left(\frac{u}{v}\right)= & \frac{\left(\Pi, u, K_{1}\left|\otimes\left(\Lambda, v, K_{2}\left|\mathcal{R}_{0}\right| \Pi, u, K_{1}\right) \otimes\right| \Lambda, v, K_{2}\right)}{\left(\varnothing, u, K_{1}\left|\otimes\left(\varnothing, v, K_{2}\left|\mathcal{R}_{0}\right| \varnothing, u, K_{1}\right) \otimes\right| \varnothing, v, K_{2}\right)}= \\
= & K_{1}^{\frac{|\Lambda|}{2}} K_{2}^{\frac{|\Pi|}{2}} \exp \left\{\sum _ { n \geq 1 } ( \frac { u } { v } ) ^ { n } \frac { 1 } { n } \left[\left(1-K_{1}^{-n}\right) \sum_{(i, j, k) \in \Lambda} q_{1}^{n i} q_{2}^{n j} q_{3}^{n k}-\left(1-K_{2}^{n}\right) \sum_{(i, j, k) \in \Pi} q_{1}^{-n i} q_{2}^{-n j} q_{3}^{-n k}+\right.\right. \\
& \left.\left.-k_{n} \sum_{(i, j, k) \in \Pi} q_{1}^{-n i} q_{2}^{-n j} q_{3}^{-n k} \sum_{(a, b, c) \in \Lambda} q_{1}^{n a} q_{2}^{n b} q_{3}^{n c}\right]\right\}= \\
= & K_{1}^{\frac{|\Lambda|}{2}} K_{2}^{\frac{|\Pi|}{2}} \prod_{(i, j, k) \in \Pi(a, b, c) \in \Lambda} \prod^{-1}\left(\frac{u}{v} q_{1}^{a-i} q_{2}^{b-j} q_{3}^{c-k}\right) \prod_{(i, j, k) \in \Pi} \frac{1-K_{2} \frac{u}{u} q_{1}^{-i} q_{2}^{-j} q_{3}^{-k}}{1-\frac{u}{v} q_{1}^{-i} q_{2}^{-j} q_{3}^{-k}} \prod_{(a, b, c) \in \Lambda} \frac{1-\frac{u}{v} q_{1}^{a} q_{2}^{b} q_{3}^{c}}{1-K_{1}^{-1} \frac{u}{v} q_{1}^{a} q_{2}^{b} q_{3}^{c}},
\end{aligned}
$$


where $G(x)=\frac{\left(1-q_{1}^{-1} x\right)\left(1-q_{2}^{-1} x\right)\left(1-q_{3}^{-1} x\right)}{\left(1-q_{1} x\right)\left(1-q_{2} x\right)\left(1-q_{3} x\right)}$. The result is a rational function, e.g.

$$
R_{[[1]] \varnothing}^{K_{1}, K_{2}}(x)=K_{2}^{\frac{1}{2}} \frac{1-x}{1-K_{2} x}, \quad R_{\varnothing[[1]]}^{K_{1}, K_{2}}(x)=K_{1}^{-\frac{1}{2}} \frac{K_{1}-x}{1-x} .
$$

It reduces to the $R$-matrix for the Fock representations in the limit of $K_{1,2} \rightarrow q_{3}=\frac{1}{q_{1} q_{2}}$.

Notice that the $R$-matrix obeys a simple inversion identity

$$
R_{\Lambda \Pi}^{K_{2}, K_{1}}(x)=\frac{1}{R_{\Pi \Lambda}^{K_{1}, K_{2}}\left(x^{-1}\right)},
$$

which does not allow one to use such an $R$-matrix for generating new knot invariants [13, section 12]. It also respects the symmetry of the DIM algebra under arbitrary permutation of parameters $q_{i}$, which needs to be accompanied by the corresponding transposition of the plane partitions $\Pi$ and $\Lambda$, e.g. exchange of $q_{i}$ and $q_{j}$ corresponds to the transposition in the $(i, j)$-plane.

In what follows, we build intertwining operators of the Fock representations with the MacMahon ones and verify that the same $R$-matrix determines their commutation relations.

\section{Intertwining operator for MacMahon representation}

The intertwiner of the quantum toroidal algebra $U_{\mathfrak{q}, \mathfrak{d}}\left(\widehat{\widehat{\mathfrak{g}}}_{1}\right)$ is the vertex operator that intertwines the tensor product of vertical and horizontal representations with an appropriate horizontal representation. Graphically, it is represented by a trivalent vertex with two "horizontal" edges and a single vertical edge. As we explained earlier, the center of $U_{\mathfrak{q}, \mathfrak{d}}\left(\widehat{\widehat{\mathfrak{g l}}}_{1}\right)$ is two dimensional, and one defines two levels $\left(\gamma_{1}, \gamma_{2}\right)$ for each representation. In the following, we fix the horizontal representation as the vertex operator representations of free deformed bosons. It has the unit first level $\gamma_{1}=\mathfrak{q}$. On the other hand, we call representations with $\gamma_{1}=1$ vertical representation, ${ }^{7}$ and there are various choices for the vertical representations, as we saw in the previous section. Note that the condition $\gamma_{1}=1$ is kept intact under taking the tensor product of two vertical representations.

In general, one can define the trivalent intertwiner $\Psi: \mathcal{V} \otimes \mathcal{H} \rightarrow \mathcal{H}^{\prime}$ for a vertical representation $\mathcal{V}$ with a pair of horizontal representations $\left(\mathcal{H}, \mathcal{H}^{\prime}\right)$ by the intertwining condition

$$
a \Psi=\Psi \Delta(a), \quad \forall a \in U_{\mathfrak{q}, \mathfrak{d}}\left(\widehat{\widehat{\mathfrak{g l}}}_{1}\right) .
$$

Because $\mathcal{V}$ has a basis $\{\alpha\}$ that simultaneously diagonalizes $K^{ \pm}(z)$, one can define the $\alpha$-component of the intertwiner as an operator between the horizontal representations

$$
\Psi_{\alpha}(\bullet)=\Psi(\alpha \otimes \bullet): \mathcal{H} \rightarrow \mathcal{H}^{\prime}, \quad \bullet \in \mathcal{H}
$$

Since $C_{1}=1$ and $C_{2}=\mathfrak{q}$ for the vertical and the horizontal representations, the definition of the coproduct (2.25)-(2.28) implies the following intertwining relations for the

\footnotetext{
${ }^{7}$ In the case of affine algebra, the evaluation representation is an example of the vertical representation.
} 
$\alpha$-components:

$$
\begin{aligned}
K^{+}(z) \Psi_{\alpha} & =\left(\alpha\left|K^{+}(z)\right| \alpha\right) \Psi_{\alpha} K^{+}(z), \\
K^{-}(\mathfrak{q} z) \Psi_{\alpha} & =\left(\alpha\left|K^{-}(z)\right| \alpha\right) \Psi_{\alpha} K^{-}(\mathfrak{q} z), \\
E(z) \Psi_{\alpha} & =\sum_{\beta}(\beta|E(z)| \alpha) \Psi_{\beta}+\left(\alpha\left|K^{-}(z)\right| \alpha\right) \Psi_{\alpha} E(z), \\
F(z) \Psi_{\alpha} & =\sum_{\beta}(\beta|F(\mathfrak{q} z)| \alpha) \Psi_{\beta} K^{+}(\mathfrak{q} z)+\Psi_{\alpha} F(z) .
\end{aligned}
$$

Note that, in the above formulas, operators at the right side of $\Psi_{\alpha}$ act on $\mathcal{H}$, while operators on the left side of $\Psi_{\alpha}$ act on $\mathcal{H}^{\prime}$. The matrix elements $(\beta|X| \alpha)$ are computed in $\mathcal{V}$. Our tasks are to construct the component of the intertwiner in the vertex operator formalism (section 2.3) and to specify admissible horizontal representations. Note that we can typically specify the relative conditions between $\mathcal{H}$ and $\mathcal{H}^{\prime}$ but there seemingly remains some freedom for each of them. At first, we will construct the vector intertwiner, which is essentially the same as the one given by [28] before. Then, as a composition of the vector intertwiner with an appropriate ordering, we will obtain the Fock intertwiner firstly given by [15]. Finally, using the same strategy we will construct the MacMahon intertwiner from the Fock intertwiner. The $N$-fold Fock intertwiner was constructed in [40], but the spectral parameters of Fock representations were independent in their construction. Our MacMahon intertwiner corresponds to an appropriate $N \rightarrow \infty$ limit of the intertwiner in [40] when the spectral parameters are correlated so that the infinite tensor product leads to the MacMahon representation.

In the following, we use the notation $\mathbf{x}(z)$ for the zero modes of the vertex operator representation $\mathcal{H}$, while $\mathbf{x}^{\prime}(z)$ for those of $\mathcal{H}^{\prime}$, where $\mathbf{x}=\mathbf{e}, \mathbf{f}, \mathbf{k}^{ \pm}$. We also denote the second level of $\mathcal{H}, \mathcal{H}^{\prime}$ by $\gamma, \gamma^{\prime}$ unless otherwise mentioned.

\subsection{Vector intertwiner}

\subsubsection{Definition of the vector intertwiner}

Let us consider the intertwining operator for the vertical vector representation $V(v)$. We define the $n$-component of the vector intertwiner by

$$
\mathbb{I}_{n}(v)(\bullet)=\mathbb{I}\left([v]_{n-1} \otimes \bullet\right): \mathcal{H} \rightarrow \mathcal{H}^{\prime}, \quad \bullet \in \mathcal{H},
$$

where $\left\{[v]_{n-1}\right\}_{n \in \mathbb{Z}}$ is the basis of $V(v)$. Explicit intertwining relations for $\mathbb{I}_{n}(v)$ are

$$
\begin{aligned}
K^{+}(z) \mathbb{I}_{n}(v) & =\tilde{\psi}\left(q_{1}^{n-1} v / z\right) \mathbb{I}_{n}(v) K^{+}(z), \\
K^{-}(\mathfrak{q} z) \mathbb{I}_{n}(v) & =\tilde{\psi}\left(q_{1}^{-n} z / v\right) \mathbb{I}_{n}(v) K^{-}(\mathfrak{q} z), \\
E(z) \mathbb{I}_{n}(v) & =\left(1-q_{2}\right) \delta\left(q_{1}^{n} v / z\right) \mathbb{I}_{n+1}(v)+\tilde{\psi}\left(q_{1}^{-n} z / v\right) \mathbb{I}_{n}(v) E(z), \\
F(z) \mathbb{I}_{n}(v) & =\left(1-q_{2}^{-1}\right) \delta\left(\mathfrak{q}^{-1} q_{1}^{n-1} v / z\right) \mathbb{I}_{n-1}(v) K^{+}(\mathfrak{q} z)+\mathbb{I}_{n}(v) F(z) .
\end{aligned}
$$

In the next subsection, we find that there are consistency conditions between the source $\mathcal{H}$ and the target $\mathcal{H}^{\prime}$, namely $\gamma^{\prime}=\gamma$ on the second level and $\mathbf{e}^{\prime}(z)=q_{2}^{-1} \mathbf{e}(z), \mathbf{f}^{\prime}(z)=q_{2} \mathbf{f}(z)$ on the zero modes. 


\subsubsection{Construction of the vector intertwiner}

We define the operator $\mathbb{I}_{n}(v)$ between two horizontal representations as

$$
\begin{aligned}
& \mathbb{I}_{n}(v)=z_{n} \tilde{\mathbb{I}}_{n}(v), \quad \tilde{\mathbb{I}}_{n}(v)=\tilde{\mathbb{I}}_{0}\left(q_{1}^{n} v\right), \quad n \in \mathbb{Z}, \\
& \tilde{\mathbb{I}}_{0}(v)=\exp \left(-\sum_{r=1}^{\infty} \frac{H_{-r}}{[r]} \frac{\left(\mathfrak{q}^{-1 / 2} v\right)^{r}}{1-q_{1}^{r}}\right) \exp \left(-\sum_{r=1}^{\infty} \frac{H_{r}}{[r]} \frac{\left(\mathfrak{q}^{1 / 2} q_{1}^{-1} v\right)^{-r}}{1-q_{1}^{r}}\right),
\end{aligned}
$$

where $z_{n}=z_{n}(v)$ is a stack of zero modes

$$
z_{0}=1, \quad z_{n}=q_{2}^{-n} \prod_{j=1}^{n} \mathbf{e}\left(q_{1}^{j-1} v\right) \quad(n>0), \quad z_{n}=q_{2}^{-n} \prod_{j=n}^{-1} \mathbf{e}\left(q_{1}^{j} v\right)^{-1} \quad(n<0) .
$$

Note that $\tilde{\mathbb{I}}_{n}(v)$ is a lift of the function (2.36) to the vertex operator, and we can formally interpret $\tilde{\mathbb{I}}_{n}(v)$ as an infinite product form

$$
: \prod_{j=n+1}^{\infty} \eta\left(q_{1}^{j-1} v\right)^{-1}:
$$

Using the fundamental OPE relations

$$
V^{+}(z) \mathbb{I}_{0}(v)=\frac{1-\mathfrak{q}^{1 / 2} q_{2} v / z}{1-\mathfrak{q}^{1 / 2} v / z}: V^{+}(z) \mathbb{I}_{0}(v):, \quad \mathbb{I}_{0}(v) V^{-}(z)=\frac{1-\mathfrak{q}^{1 / 2} q_{1} z / v}{1-\mathfrak{q}^{1 / 2} q_{3}^{-1} z / v}: \mathbb{I}_{0}(v) V^{-}(z):,
$$

one can check the following relations for the zero-component $\mathbb{I}_{0}(v)$ :

$$
\begin{aligned}
\varphi^{+}\left(\mathfrak{q}^{-1 / 2} z\right) \mathbb{I}_{0}(v) & =\tilde{\psi}\left(q_{1}^{-1} v / z\right) \mathbb{I}_{0}(v) \varphi^{+}\left(\mathfrak{q}^{-1 / 2} z\right) \\
\mathbb{I}_{0}(v) \varphi^{-}\left(\mathfrak{q}^{1 / 2} z\right) & =\tilde{\psi}(z / v)^{-1} \varphi^{-}\left(\mathfrak{q}^{1 / 2} z\right) \mathbb{I}_{0}(v), \\
\xi(z) \mathbb{I}_{0}(v) & =\frac{1-\mathfrak{q} v / z}{1-\mathfrak{q} q_{2} v / z}: \xi(z) \mathbb{I}_{0}(v):, \quad \mathbb{I}_{0}(v) \xi(z)=\frac{1-\mathfrak{q} q_{3}^{-1} z / v}{1-\mathfrak{q} q_{1} z / v}: \mathbb{I}_{0}(v) \xi(z): \\
\eta(z) \mathbb{I}_{0}(v) & =\frac{1-q_{2} v / z}{1-v / z}: \eta(z) \mathbb{I}_{0}(v):, \quad \mathbb{I}_{0}(v) \eta(z)=\frac{1-q_{1} z / v}{1-q_{3}^{-1} z / v}: \mathbb{I}_{0}(v) \eta(z):
\end{aligned}
$$

Actually, we have defined $\mathbb{I}_{0}(v)$ so that it satisfies the relations (4.17), (4.18), which imply $\gamma=\gamma^{\prime}$ on the second level. The relative relation for the zero modes is determined from the intertwining relations for $F(z)$ and $E(z)$. To see it, let us check the intertwining relation for the 0-component for $F(z)$. To obtain the relation (4.11), we find that the additional factor $q_{2}$ of $F(z)$ on the left is necessary, compared to $F(z)$ on the right, namely

$$
q_{2} \xi(z) \mathbf{f}(z) \mathbb{I}_{0}(v)-\mathbb{I}_{0}(v) \xi(z) \mathbf{f}(z)=\left(1-q_{2}^{-1}\right) \delta\left(\frac{q_{1}^{-1} v}{\mathfrak{q} z}\right) q_{2} \mathbf{e}\left(q_{1}^{-1} v\right)^{-1} \tilde{\mathbb{I}}_{-1}(v) \varphi^{+}\left(\mathfrak{q}^{1 / 2} z\right) \gamma^{-1},
$$


where we have used the relation (2.91) and

$$
\delta\left(\frac{q_{1}^{-1} v}{\mathfrak{q} z}\right) \xi(z)=\delta\left(\frac{q_{1}^{-1} v}{\mathfrak{q} z}\right) \eta\left(q_{1}^{-1} v\right)^{-1} \varphi^{+}\left(\mathfrak{q}^{1 / 2} z\right)
$$

This is the reason why the vector intertwiner should shift the horizontal zero mode by $q_{2}^{-1}$. In the same way, one can check

$$
q_{2}^{-1} \eta(z) \mathbf{e}(z) \mathbb{I}_{0}(v)-\tilde{\psi}(z / v) \mathbb{I}_{0}(v) \eta(z) \mathbf{e}(z)=\left(1-q_{2}\right) \delta(v / z) q_{2}^{-1} \mathbf{e}(v) \tilde{\mathbb{I}}_{1}(v)
$$

The intertwining relation for general components can be also checked easily by making use of the relation among the functions $G(z), \tilde{\psi}(z)$ and $s(z)$.

If we choose the horizontal representation $\mathcal{H}=\mathcal{F}_{u}^{\left(\mathfrak{q}, \mathfrak{q}^{N}\right)}$ used in [15], the zero mode sector is defined by $(2.94)$ and $\mathcal{H}^{\prime}=\mathcal{F}_{q_{2}^{-1} u}^{\left(\mathfrak{q}, \mathfrak{q}^{N}\right)}$. Then, the zero mode stack $z_{n}=z_{n}(N ; u \mid v)$ is

$$
z_{n}=\left(u / q_{2}\right)^{n} \prod_{j=1}^{n}\left(\frac{\mathfrak{q}}{q_{1}^{j-1} v}\right)^{N} \quad(n>0), \quad z_{n}=\left(u / q_{2}\right)^{n} \prod_{j=n}^{-1}\left(\frac{\mathfrak{q}}{q_{1}^{j-1} v}\right)^{N} \quad(n<0) .
$$

Essentially the same vector intertwiner was given in the Appendix of [28], where they computed the generating function of the components of the intertwiner (4.7). By the simple $n$-dependence (4.12) of the components, it is easy to see that the intertwiner in [28] agrees with ours.

\subsection{Fock intertwiner}

\subsubsection{Definition of the Fock intertwiner}

Let us consider the intertwining operator for the vertical Fock representation $\mathcal{F}(v)$. With the basis $\{\mid \lambda)\}$ of $\mathcal{F}(v)$, explicit intertwining relations for the $\lambda$-component $\Phi_{\lambda}(v)$ are

$$
\begin{aligned}
K^{+}(z) \Phi_{\lambda}(v) & =\left(\lambda\left|K^{+}(z)\right| \lambda\right) \Phi_{\lambda}(v) K^{+}(z), \\
K^{-}(\mathfrak{q} z) \Phi_{\lambda}(v) & =\left(\lambda\left|K^{-}(z)\right| \lambda\right) \Phi_{\lambda}(v) K^{-}(\mathfrak{q} z), \\
E(z) \Phi_{\lambda}(v) & =\sum_{k=1}^{\ell(\lambda)+1}\left(\lambda+1_{k}|E(z)| \lambda\right) \Phi_{\lambda+1_{k}}(v)+\left(\lambda\left|K^{-}(z)\right| \lambda\right) \Phi_{\lambda}(v) E(z), \\
F(z) \Phi_{\lambda}(v) & =\sum_{k=1}^{\ell(\lambda)}\left(\lambda-1_{k}|F(\mathfrak{q} z)| \lambda\right) \Phi_{\lambda-1_{k}}(v) K^{+}(\mathfrak{q} z)+\Phi_{\lambda}(v) F(z),
\end{aligned}
$$

where matrix elements can be read from (2.57)-(2.60). In the next subsection, we obtain as consistency conditions the relative shift $\gamma^{\prime}=\mathfrak{q} \gamma$ of the second level and $\mathbf{e}^{\prime}(z)=(-\mathfrak{q} v / z) \mathbf{e}(z), \mathbf{f}^{\prime}(z)=(-\mathfrak{q} v / z)^{-1} \mathbf{f}(z)$ on the relative shift of the zero modes. 


\subsubsection{Construction of the Fock intertwiner}

We construct the Fock intertwiner in a parallel way to the construction of the Fock representation (section 2.2.2) in contrast to a rather direct way of [15]. In our approach, taking the tensor product of the vertical representations is realized by merely composing the corresponding vector intertwiners. And the modification factor $\beta_{n}^{ \pm}(v / z)$ is lifted to the corresponding vertex operator $B_{n}(v)$.

We begin with specifying the modification operator $B_{n}(v)$. This is realized by making use of the dual vertex operator (2.97) as

$$
B_{n}(v)=\tilde{V}^{-}\left(\mathfrak{q}^{1 / 2} q_{2}^{n} v\right) \tilde{V}^{+}\left(\mathfrak{q}^{3 / 2} q_{2}^{n} v\right)^{-1}
$$

and satisfies the following relations

$$
\begin{aligned}
\varphi^{+}\left(\mathfrak{q}^{-1 / 2} z\right) B_{n}(v) & =\mathfrak{q} \beta_{n}^{+}(v / z) B_{n}(v) \varphi^{+}\left(\mathfrak{q}^{-1 / 2} z\right), \\
B_{n}(v) \varphi^{-}\left(\mathfrak{q}^{1 / 2} z\right) & =\mathfrak{q} \beta_{n}^{-}(z / v)^{-1} \varphi^{-}\left(\mathfrak{q}^{1 / 2} z\right) B_{n}(v), \\
\left(\frac{\mathfrak{q}}{z}\left(-q_{2}^{n} v\right)\right)^{-1} \xi(z) B_{n}(v)-B_{n}(v) \xi(z) & =0, \\
\frac{\mathfrak{q}}{z}\left(-q_{2}^{n} v\right) \eta(z) B_{n}(v)-\beta_{n}^{-}(z / v) B_{n}(v) \eta(z) & =-\mathfrak{q} \delta\left(q_{2}^{n} v / z\right): \eta(z) B_{n}(v): .
\end{aligned}
$$

Note that we can formally interpret $B_{n}(v)$ as an infinite product form

$$
: \prod_{i, j=1}^{\infty} \eta\left(q_{1}^{j-1} q_{2}^{n+i-1} v\right)^{-1}: .
$$

(4.30) and (4.31) mean that the operator $B_{n}(v)$ exactly corresponds to the modification factor $\beta_{n}^{ \pm}(v / z)$ up to the monomial factor $\mathfrak{q}$, and this discrepancy leads to the relative level shift. Furthermore, (4.32) and (4.33) lead to the relative shift of the zero modes. We can summarize the conditions for $B_{n}(v): \mathcal{H} \rightarrow \mathcal{H}^{\prime \prime}$ as follows

$$
\gamma^{\prime \prime}=\mathfrak{q} \gamma, \quad \mathbf{e}^{\prime \prime}(z)=\left(-\mathfrak{q} q_{2}^{n} v / z\right) \mathbf{e}(z), \quad \mathbf{f}^{\prime \prime}(z)=\left(-\mathfrak{q} q_{2}^{n} v / z\right)^{-1} \mathbf{f}(z) .
$$

Now we can define the operator $\Phi_{\lambda}(v)$ between two horizontal representations as the following composition ${ }^{8}$ of vector intertwiners with the modification operator $B_{n}(v)$

$$
\begin{aligned}
& \Phi_{\lambda}(v)=z_{\lambda} \tilde{\Phi}_{\lambda}(v): \mathcal{H} \rightarrow \mathcal{H}^{\prime \prime} \rightarrow \mathcal{H}^{\prime}, \\
& \tilde{\Phi}_{\lambda}(v)=\mathcal{G}^{[n]} \cdot \tilde{\mathbb{I}}_{\lambda}^{[n]}(v) B_{n}(v), \quad n>\ell(\lambda),
\end{aligned}
$$

where $\tilde{\mathbb{I}}_{\lambda}^{[n]}(v)=\tilde{\mathbb{I}}_{\lambda_{1}}(v) \circ \cdots \circ \tilde{\mathbb{I}}_{\lambda_{n}}\left(q_{2}^{n-1} v\right)$ and the coefficient $\mathcal{G}^{[n]}$ is defined by the normal ordering $^{9}$

$$
\tilde{\mathbb{I}}_{\varnothing}^{[n]}(v) B_{n}(v)=\left(\mathcal{G}^{[n]}\right)^{-1}: \tilde{\mathbb{I}}_{\varnothing}^{[n]}(v) B_{n}(v): .
$$

${ }^{8}$ One should be careful of the ordering of constituent vector intertwiners.

${ }^{9}$ We have defined as $\lambda_{n}=0$ for $n>\ell(\lambda)$. 
Finally, $z_{\lambda}=z_{\lambda}(v)$ is a stack of zero modes (see the discussion of the intertwiner zero modes in appendix $\mathrm{C}$ )

$$
z_{\lambda}(v)=\prod_{i=1}^{\ell(\lambda)} \prod_{j=1}^{\lambda_{i}}\left(-\mathfrak{q} q_{2}^{i-1} x_{i, j}^{-1}\right) \mathbf{e}\left(x_{i, j} v\right)=q_{2}^{n(\lambda)}(-\mathfrak{q})^{|\lambda|} \prod_{(i, j) \in \lambda} x_{i, j}^{-1} \mathbf{e}\left(x_{i, j} v\right) .
$$

Note that the definition (4.37) is independent of $n>\ell(\lambda)$ thanks to the relation

$$
\begin{aligned}
: \tilde{\mathbb{I}}_{\lambda}^{[n]}(v) B_{n}(v):= & : \tilde{\mathbb{I}}_{\lambda}^{n n+1]}(v) B_{n+1}(v): \\
= & \exp \left(\sum_{r=1}^{\infty} \frac{H_{-r}}{[r]}\left(\mathfrak{q}^{-1 / 2} v\right)^{r}\left(\sum_{(i, j) \in \lambda} x_{i, j}^{r}-\frac{1}{\left(1-q_{1}^{r}\right)\left(1-q_{2}^{r}\right)}\right)\right) \\
& \times \exp \left(-\sum_{r=1}^{\infty} \frac{H_{r}}{[r]}\left(\mathfrak{q}^{1 / 2} v\right)^{-r}\left(\sum_{(i, j) \in \lambda} x_{i, j}^{-r}-\frac{q_{3}^{-r}}{\left(1-q_{1}^{r}\right)\left(1-q_{2}^{r}\right)}\right)\right),
\end{aligned}
$$

which can be also understood from the formal infinite product form of them. The consistency conditions between the horizontal representations in $\Phi_{\lambda}(v): \mathcal{H} \rightarrow \mathcal{H}^{\prime}$ can be read as

$$
\gamma^{\prime}=\mathfrak{q} \gamma, \quad \mathbf{e}^{\prime}(z)=(-\mathfrak{q} v / z) \mathbf{e}(z), \quad \mathbf{f}^{\prime}(z)=(-\mathfrak{q} v / z)^{-1} \mathbf{f}(z)
$$

Indeed, these zero modes of $\mathcal{H}^{\prime}$ satisfy the relations (2.91). We can confirm that the operator $\Phi_{\lambda}(v)$ agrees with the AFS intertwiner [15], and the factor $\mathcal{G}_{\lambda}$, which is defined independently of $n>\ell(\lambda)$ by

$$
\tilde{\mathbb{I}}_{\lambda}^{[n]}(v) B_{n}(v)=\left(\mathcal{G}_{\lambda}\right)^{-1}\left(\mathcal{G}^{[n]}\right)^{-1}: \tilde{\mathbb{I}}_{\lambda}^{[n]}(v) B_{n}(v):,
$$

plays the same role as $c_{\lambda}$ in [15]. An explicit form of $\mathcal{G}_{\lambda}$ is

$$
\begin{aligned}
\mathcal{G}_{\lambda} & =\prod_{\square \in \lambda}\left(1-q_{1}^{-a_{\lambda}(\square)} q_{2}^{l_{\lambda}(\square)+1}\right)=q_{1}^{-n\left(\lambda^{\prime}\right)} q_{2}^{n(\lambda)+|\lambda|} c_{\lambda}, \\
c_{\lambda} & =\prod_{\square \in \lambda}\left(1-q_{1}^{a_{\lambda}(\square)} q_{2}^{-l_{\lambda}(\square)-1}\right) .
\end{aligned}
$$

If we choose the AFS type horizontal representation $\mathcal{H}=\mathcal{F}_{u}^{\left(\mathfrak{q}, \mathfrak{q}^{N}\right)}$, where the zero mode action is defined by $(2.94)$, then $\mathcal{H}^{\prime}=\mathcal{F}_{-v u}^{\left(\mathfrak{q}, \mathfrak{q}^{N+1}\right)}$, and the zero mode stack $z_{\lambda}=z_{\lambda}(N ; u \mid v)$ is

$$
\begin{aligned}
z_{\lambda}(N ; u \mid v) & =\prod_{i=1}^{\ell(\lambda)} z_{\lambda_{i}}\left(N+1 ;-q_{2}^{i} v u \mid q_{2}^{i-1} v\right)=\prod_{i=1}^{\ell(\lambda)}\left(-q_{2}^{i-1} v u\right)^{\lambda_{i}} \prod_{j=1}^{\lambda_{i}}\left(\frac{\mathfrak{q}}{q_{1}^{j-1} q_{2}^{i-1} v}\right)^{N+1} \\
& =q_{2}^{n(\lambda)}(-v u)^{|\lambda|} \prod_{(i, j) \in \lambda}\left(\mathfrak{q} x_{i, j}^{-1} v^{-1}\right)^{N+1}
\end{aligned}
$$

where $n(\lambda)=\sum_{j=1}^{\ell(\lambda)}(j-1) \lambda_{j}$ and $x_{i, j}=q_{1}^{j-1} q_{2}^{i-1}$. 
One can check the Fock intertwining relations by making use of the vector intertwining relation. For example, to check the intertwining relation with $E(z)$, one can compute as follows:

$$
\begin{aligned}
& E(z) \Phi_{\lambda}(v)- \prod_{s=1}^{n} \tilde{\psi}\left(q_{1}^{-\lambda_{s}} q_{2}^{1-s} z / v\right) \beta_{n}^{-}(z / v) \Phi_{\lambda}(v) E(z) \\
&=z_{\lambda} \mathcal{G}^{[n]} \sum_{k=1}^{n}\left(-\mathfrak{q} q_{2}^{k} v / z\right) \prod_{s=1}^{k-1} \tilde{\psi}\left(q_{1}^{-\lambda_{s}} q_{2}^{1-s} z / v\right) \tilde{\mathbb{I}}_{\lambda_{1}}(v) \cdots \tilde{\mathbb{I}}_{\lambda_{k-1}}\left(q_{2}^{k-2} v\right) \\
& \quad \times\left[\left[E(z), \tilde{\mathbb{I}}_{\lambda_{k}}\left(q_{2}^{k-1} v\right)\right]\right]_{k} \tilde{\mathbb{I}}_{\lambda_{k+1}}\left(q_{2}^{k} v\right) \cdots \tilde{\mathbb{I}}_{\lambda_{n}}\left(q_{2}^{n-1} v\right) B_{n}(v) \\
& \quad+z_{\lambda} \mathcal{G}^{[n]} \prod_{s=1}^{n} \tilde{\psi}\left(q_{1}^{-\lambda_{s}} q_{2}^{1-s} z / v\right) \tilde{\mathbb{I}}_{\lambda}^{[n]}(v)\left(E(z) B_{n}(v)-\beta^{-}(z / v) B_{n}(v) E(z)\right),
\end{aligned}
$$

where we have introduced the "weighted" commutator by

$$
[[A, B]]_{k}=A B-\tilde{\psi}\left(q_{1}^{-\lambda_{k}} q_{2}^{1-k} z / v\right) B A
$$

and the zero mode $\mathbf{e}(z)$ of $E(z)$ should be adjusted appropriately according to the space on which $E(z)$ acts. First of all, the last term on the r.h.s. vanishes due to the factor $\tilde{\psi}\left(q_{2}^{-n+1} z / v\right) \delta\left(q_{2}^{n} v / z\right)$. The intertwining relation for the vector intertwiner tells us that

$$
\left[\left[E(z), \tilde{\mathbb{I}}_{\lambda_{k}}\left(q_{2}^{k-1} v\right)\right]\right]_{k}=\left(1-q_{2}\right) \delta\left(q_{1} x_{k} v / z\right) q_{2}^{-1} \mathbf{e}(z) \tilde{\mathbb{I}}_{\lambda_{k}+1}\left(q_{2}^{k-1} v\right) .
$$

Hence, we obtain

$$
\begin{aligned}
& E(z) \Phi_{\lambda}(v)-\left(\lambda\left|K^{-}(z)\right| \lambda\right) \Phi_{\lambda}(v) E(z) \\
& =\left(1-q_{2}\right) z_{\lambda} \mathcal{G}^{[n]} \cdot q_{2}^{-1} \sum_{k=1}^{n}\left(-\mathfrak{q} q_{2}^{k} v / z\right) \prod_{s=1}^{k-1} \tilde{\psi}\left(q_{1}^{-\lambda_{s}} q_{2}^{1-s} z / v\right) \tilde{\mathbb{I}}_{\lambda_{1}}(v) \cdots \tilde{\mathbb{I}}_{\lambda_{k-1}}\left(q_{2}^{k-2} v\right) \\
& \quad \quad \quad \delta\left(q_{1} x_{k} v / z\right) \mathbf{e}(z) \tilde{\mathbb{I}}_{\lambda_{k}+1}\left(q_{2}^{k-1} v\right) \tilde{\mathbb{I}}_{\lambda_{k+1}}\left(q_{2}^{k} v\right) \cdots \tilde{\mathbb{I}}_{\lambda_{n}}\left(q_{2}^{n-1} v\right) B_{n}(v) \\
& =\sum_{k=1}^{n}\left(\lambda_{k}+1|E(z)| \lambda\right) \Phi_{\lambda+1_{k}}(v) .
\end{aligned}
$$

As concerns the intertwining relation with $F(z)$, one can compute as follows:

$$
\begin{aligned}
F(z) \Phi_{\lambda}(v)-\Phi_{\lambda}(v) F(z)= & z_{\lambda} \mathcal{G}^{[n]} \sum_{k=1}^{n}\left(-\mathfrak{q} q_{2}^{k} v / z\right)^{-1} \tilde{\mathbb{I}}_{\lambda_{1}}(v) \cdots \tilde{\mathbb{I}}_{\lambda_{k-1}}\left(q_{2}^{k-2} v\right) \\
& \times\left[F(z), \tilde{\mathbb{I}}_{\lambda_{k}}\left(q_{2}^{k-1} v\right)\right] \tilde{\mathbb{I}}_{\lambda_{k+1}}\left(q_{2}^{k} v\right) \cdots \tilde{\mathbb{I}}_{\lambda_{n}}\left(q_{2}^{n-1} v\right) B_{n}(v)+z_{\lambda} \mathcal{G}^{[n]} \cdot \tilde{\mathbb{I}}_{\lambda}^{[n]}(v)\left[F(z), B_{n}(v)\right] .
\end{aligned}
$$

Note that this time we do not have to use the "weighted" commutator. Again, the last term vanishes and, by substituting the intertwining relations for the vector intertwiner, we 
obtain

$$
\begin{aligned}
& F(z) \Phi_{\lambda}(v)-\Phi_{\lambda}(v) F(z) \\
& =\left(1-q_{2}^{-1}\right) z_{\lambda} \mathcal{G}^{[n]} \cdot q_{2} \sum_{k=1}^{n}\left(-\mathfrak{q} q_{2}^{k} v / z\right)^{-1} \tilde{\mathbb{I}}_{\lambda_{1}}(v) \cdots \tilde{\mathbb{I}}_{\lambda_{k-1}}\left(q_{2}^{k-2} v\right) \\
& \quad \times \delta\left(\mathfrak{q}^{-1} x_{k} v / z\right) \mathbf{f}(z) \tilde{\mathbb{I}}_{\lambda_{k}-1}\left(q_{2}^{k-1} v\right) K^{+}(\mathfrak{q} z) \tilde{\mathbb{I}}_{\lambda_{k+1}}\left(q_{2}^{k} v\right) \cdots \tilde{\mathbb{I}}_{\lambda_{n}}\left(q_{2}^{n-1} v\right) B_{n}(v) \\
& =\left(1-q_{2}^{-1}\right) z_{\lambda} \mathcal{G}^{[n]} \cdot q_{2} \sum_{k=1}^{n}\left(-\mathfrak{q} q_{2}^{k} v / z\right)^{-1} \prod_{s=k+1}^{n} \tilde{\psi}\left(q_{1}^{\lambda_{s}-1} q_{2}^{s-1} v / z\right) \beta_{n}^{+}(v / z) \tilde{\mathbb{I}}_{\lambda_{1}}(v) \cdots \tilde{\mathbb{I}}_{\lambda_{k-1}}\left(q_{2}^{k-2} v\right) \\
& \quad \times \delta\left(\mathfrak{q}^{-1} x_{k} v / z\right) \mathbf{f}(z) \tilde{\mathbb{I}}_{\lambda_{k}-1}\left(q_{2}^{k-1} v\right) \tilde{\mathbb{I}}_{\lambda_{k+1}}\left(q_{2}^{k} v\right) \cdots \tilde{\mathbb{I}}_{\lambda_{n}}\left(q_{2}^{n-1} v\right) B_{n}(v) K^{+}(\mathfrak{q} z) \\
& =\sum_{k=1}^{n}\left(\lambda_{k}-1|F(z)| \lambda\right) \Phi_{\lambda-1_{k}}(v) K^{+}(\mathfrak{q} z) .
\end{aligned}
$$

\subsection{MacMahon intertwiner}

\subsubsection{Definition of the MacMahon intertwiner}

Let us consider the intertwining operator for the vertical MacMahon representation $\mathcal{M}(K ; v)$. We define the MacMahon intertwiner $\Xi(K ; v)$ by the following condition

$$
\Xi(K ; v): \mathcal{M}(K ; v) \otimes \mathcal{H} \rightarrow \mathcal{H}^{\prime}, \quad a \Psi=\Psi \Delta(a), \quad a \in U_{\mathfrak{q}, \mathfrak{d}}\left(\widehat{\widehat{g l}}_{1}\right),
$$

and the $\Lambda$-component of the MacMahon intertwiner by

$$
\left.\Xi_{\Lambda}(\bullet)=\Xi(\mid \Lambda) \otimes \bullet\right): \mathcal{H} \rightarrow \mathcal{H}^{\prime}, \quad \bullet \in \mathcal{H},
$$

where $\{\mid \Lambda)\}$ is the basis of $\mathcal{M}(K ; v)$. Explicit intertwining relations for $\Xi_{\Lambda}(K ; v)$ are

$$
\begin{aligned}
K^{+}(z) \Xi_{\Lambda}(v) & =\left(\Lambda\left|K^{+}(z)\right| \Lambda\right) \Xi_{\Lambda}(v) K^{+}(z), \\
K^{-}(\mathfrak{q} z) \Xi_{\Lambda}(v) & =\left(\Lambda\left|K^{-}(z)\right| \Lambda\right) \Xi_{\Lambda}(v) K^{-}(\mathfrak{q} z), \\
E(z) \Xi_{\Lambda}(v) & =\sum\left(\Lambda+1_{i}^{(k)}|E(z)| \Lambda\right) \Xi_{\Lambda+1_{i}^{(k)}}(v)+\left(\Lambda\left|K^{-}(z)\right| \Lambda\right) \Xi_{\Lambda}(v) E(z), \\
F(z) \Xi_{\Lambda}(v) & =\sum\left(\Lambda-1_{i}^{(k)}|F(\mathfrak{q} z)| \Lambda\right) \Xi_{\Lambda-1_{i}^{(k)}}(v) K^{+}(\mathfrak{q} z)+\Xi_{\Lambda}(v) F(z),
\end{aligned}
$$

where the matrix elements can be read from (2.75)-(2.77). In the next subsection, we specify the constraint on the relations among the zero modes.

\subsubsection{Construction of the MacMahon intertwiner}

We construct the MacMahon intertwiner in the same way as the construction of the Fock intertwiner in section 4.2. We begin with specifying the vertex operator $\Gamma_{n}(K ; v)$ that produces the modification factor $\gamma_{n}^{ \pm}(z / v)$ given by (2.74) in the OPE relation. This operator is realized by

$$
\Gamma_{n}(K ; v)=\exp \left(\sum_{r=1}^{\infty} \frac{H_{-r}}{[r]} \frac{q_{3}^{n r}-K^{r}}{k_{r}}\left(\mathfrak{q}^{-1 / 2} v\right)^{r}\right) \exp \left(\sum_{r=1}^{\infty} \frac{H_{r}}{[r]} \frac{q_{3}^{-n r}-K^{-r}}{k_{r}}\left(\mathfrak{q}^{1 / 2} v\right)^{-r}\right),
$$


and satisfies the following fundamental relations

$$
\begin{aligned}
& V^{+}(z) \Gamma_{n}(K ; v)=\exp \left(\sum_{r=1}^{\infty} \frac{1}{r} \frac{q_{3}^{n r}-K^{r}}{1-q_{3}^{r}}\left(\mathfrak{q}^{1 / 2} v / z\right)^{r}\right): V^{+}(z) \Gamma_{n}(K ; v): \\
& \Gamma_{n}(K ; v) V^{-}(z)=\exp \left(-\sum_{r=1}^{\infty} \frac{1}{r} \frac{q_{3}^{-n r}-K^{-r}}{1-q_{3}^{r}}\left(\mathfrak{q}^{3 / 2} v / z\right)^{-r}\right): \Gamma_{n}(K ; v) V^{-}(z): .
\end{aligned}
$$

Note that we can formally interpret $\Gamma_{n}(K ; v)$ as an infinite product form as

$$
: \prod_{i, j, k=1}^{\infty} \eta\left(q_{1}^{j-1} q_{2}^{i-1} q_{3}^{n+k-1} v\right)^{-1} \eta\left(q_{1}^{j-1} q_{2}^{i-1} q_{3}^{k-1} K v\right):
$$

Then we find the following relations:

$$
\begin{aligned}
\varphi^{+}\left(\mathfrak{q}^{-1 / 2} z\right) \Gamma_{n}(K ; v) & =\frac{K^{1 / 2}}{\mathfrak{q}^{n}} \gamma_{n}^{+}(v / z) \Gamma_{n}(K ; v) \varphi^{+}\left(\mathfrak{q}^{-1 / 2} z\right), \\
\Gamma_{n}(K ; v) \varphi^{-}\left(\mathfrak{q}^{1 / 2} z\right) & =\frac{K^{1 / 2}}{\mathfrak{q}^{n}} \gamma_{n}^{-}(z / v)^{-1} \varphi^{-}\left(\mathfrak{q}^{1 / 2} z\right) \Gamma_{n}(K ; v),
\end{aligned}
$$

which mean that the operator $\Gamma_{n}(K ; v)$ produces the modification factor $\gamma_{n}^{ \pm}(v / z)$ up to a monomial factor. This discrepancy corresponds to a shift of the second level:

$$
\gamma^{\prime \prime}=\mathfrak{q}^{-n} K^{1 / 2} \gamma
$$

Furthermore, the normal orderings with $\xi$ and $\eta$ are evaluated to give

$$
\begin{aligned}
& \xi(z) \Gamma_{n}(K ; v)=\frac{\left(\mathfrak{q} q_{3}^{n} v / z ; q_{3}\right)_{\infty}}{\left(K \mathfrak{q} v / z ; q_{3}\right)_{\infty}}: \xi(z) \Gamma_{n}(K ; v):, \\
& \Gamma_{n}(K ; v) \xi(z)=\frac{\left(K^{-1} \mathfrak{q} z / v ; q_{3}\right)_{\infty}}{\left(\mathfrak{q} q_{3}^{-n} z / v ; q_{3}\right)_{\infty}}: \xi(z) \Gamma_{n}(K ; v):, \\
& \eta(z) \Gamma_{n}(K ; v)=\frac{\left(K v / z ; q_{3}\right)_{\infty}}{\left(q_{3}^{n} v / z ; q_{3}\right)_{\infty}}: \eta(z) \Gamma_{n}(K ; v):, \\
& \Gamma_{n}(K ; v) \eta(z)=\frac{\left(q_{3}^{-n} z / v ; q_{3}\right)_{\infty}}{\left(K^{-1} z / v ; q_{3}\right)_{\infty}}: \eta(z) \Gamma_{n}(K ; v): .
\end{aligned}
$$

In order to obtain the relation $F(z) \Gamma_{n}(K ; v)-\Gamma_{n}(K ; v) F(z)=0$, we need the relative shift

$$
\left[\frac{\left(\mathfrak{q} q_{3}^{n} v / z ; q_{3}\right)_{\infty}}{\left(K \mathfrak{q} v / z ; q_{3}\right)_{\infty}}\right]^{-1} \frac{\left(K^{-1} \mathfrak{q} z / v ; q_{3}\right)_{\infty}}{\left(\mathfrak{q} q_{3}^{-n} z / v ; q_{3}\right)_{\infty}}=\frac{\theta_{q_{3}}(\mathfrak{q} K v / z)}{\theta_{q_{3}}\left(\mathfrak{q} q_{3}^{n} v / z\right)},
$$

where the theta function $\theta_{q_{3}}(z)$ is defined by (B.1). Note that this in turn determines the relative shift for $\mathbf{e}(z)$. As a result, we have

$$
\begin{array}{r}
\frac{\theta_{q_{3}}(\mathfrak{q} K v / z)}{\theta_{q_{3}}\left(\mathfrak{q} q_{3}^{n} v / z\right)} \xi(z) \Gamma_{n}(K ; v)-\Gamma_{n}(K ; v) \xi(z)=0, \\
\frac{K^{1 / 2}}{\mathfrak{q}^{n}} \frac{\theta_{q_{3}}\left(q_{3}^{n} v / z\right)}{\theta_{q_{3}}(K v / z)} \eta(z) \Gamma_{n}(K ; v)-\gamma_{n}^{-}(z / v) \Gamma_{n}(K ; v) \eta(z)=0 .
\end{array}
$$


Note that (4.71) vanishes by itself, while the corresponding equation (4.33) vanishes only after multiplying with the factor $\tilde{\psi}\left(q_{2}^{-n+1} z / v\right)$. We can summarize the relative condition for $\Gamma_{n}(K ; v): \mathcal{H} \rightarrow \mathcal{H}^{\prime \prime}$ as follows

$$
\mathbf{e}^{\prime \prime}(z)=\frac{K^{1 / 2}}{\mathfrak{q}^{n}} \frac{\theta_{q_{3}}\left(q_{3}^{n} v / z\right)}{\theta_{q_{3}}(K v / z)} \mathbf{e}(z), \quad \mathbf{f}^{\prime \prime}(z)=\frac{\theta_{q_{3}}(\mathfrak{q} K v / z)}{\theta_{q_{3}}\left(\mathfrak{q} q_{3}^{n} v / z\right)} \mathbf{f}(z) .
$$

Now we can define the operator $\Xi_{\Lambda}(K ; v)$ between two horizontal representations as a well-ordered stack of the Fock intertwiners with the vertex operator $\Gamma_{n}(K ; v)$

$$
\begin{aligned}
& \Xi_{\Lambda}(K ; v)=z_{\Lambda} \tilde{\Xi}_{\Lambda}(K ; v): \mathcal{H} \rightarrow \mathcal{H}^{\prime \prime} \rightarrow \mathcal{H}^{\prime}, \\
& \tilde{\Xi}_{\Lambda}(K ; v)=\mathcal{M}^{[n]}(K) \tilde{\Phi}_{\Lambda}^{[n]}(v) \Gamma_{n}(K ; v), \quad n>h(\Lambda),
\end{aligned}
$$

where the factor $\mathcal{M}^{[n]}(K)$ is defined as ${ }^{10}$

$$
\begin{aligned}
\tilde{\Phi}_{\varnothing}^{[n]}(v) \Gamma_{n}(K ; v) & =\mathcal{M}^{[n]}(K)^{-1}: \tilde{\Phi}_{\varnothing}^{[n]}(v) \Gamma_{n}(K ; v):, \\
\tilde{\Phi}_{\Lambda}^{[n]}(v) & =\tilde{\Phi}_{\Lambda^{(1)}}(v) \circ \cdots \circ \tilde{\Phi}_{\Lambda^{(n)}}\left(q_{3}^{n-1} v\right),
\end{aligned}
$$

and $z_{\Lambda}=z_{\Lambda}(K ; v)$ is the contribution from the zero modes,

$$
z_{\Lambda}(K ; v)=\prod_{k=1}^{h(\Lambda)} \prod_{(i, j) \in \Lambda^{(k)}} \frac{K^{1 / 2}}{\mathfrak{q}^{k-1}} \frac{\theta_{q_{3}}\left(q_{3}^{k-1} / x_{i j k}\right)}{\theta_{q_{3}}\left(K / x_{i j k}\right)} \mathbf{e}\left(x_{i j k} v\right) .
$$

Note that the definition (4.74) is independent of $n>h(\Lambda)$ thanks to the relation

$$
\begin{aligned}
&: \tilde{\Phi}_{\Lambda}^{[n]}(v) \Gamma_{n}(K ; v):=: \tilde{\Phi}_{\Lambda}^{[n+1]}(v) \Gamma_{n+1}(K ; v): \\
&=\exp \left(\sum_{r=1}^{\infty} \frac{H_{-r}}{[r]}\left(\mathfrak{q}^{-1 / 2} v\right)^{r}\left(\sum_{(i, j, k) \in \Lambda} x_{i j k}^{r}+\frac{1-K^{r}}{k_{r}}\right)\right) \\
& \quad \times \exp \left(-\sum_{r=1}^{\infty} \frac{H_{r}}{[r]}\left(\mathfrak{q}^{1 / 2} v\right)^{-r}\left(\sum_{(i, j, k) \in \Lambda} x_{i j k}^{-r}-\frac{1-K^{-r}}{k_{r}}\right)\right),
\end{aligned}
$$

which can be also understood from the formal infinite product form of them. The relative condition of horizontal representations for $\Xi_{\Lambda}(K ; v): \mathcal{H} \rightarrow \mathcal{H}^{\prime}$ can be read as

$$
\gamma^{\prime}=K^{1 / 2} \gamma, \quad \mathbf{e}^{\prime}(z)=K^{1 / 2} \frac{\theta_{q_{3}}(v / z)}{\theta_{q_{3}}(K v / z)} \mathbf{e}(z), \quad \mathbf{f}^{\prime}(z)=\frac{\theta_{q_{3}}(\mathfrak{q} K v / z)}{\theta_{q_{3}}(\mathfrak{q} v / z)} \mathbf{f}(z),
$$

where we have used the formula in appendix B. Indeed, these zero modes of $\mathcal{H}^{\prime}$ satisfy the relations (2.91) as long as those of $\mathcal{H}$ satisfy the relations (2.91). Hence, the operator $\Xi_{\Lambda}(K ; v)$ exists for each horizontal representation $\mathcal{H}$.

The intertwining relations for $\Xi_{\Lambda}(K ; v)$ essentially follow from those for the Fock intertwiners. A proof can be done almost in parallel with the computations we have shown in the check of the intertwining relations for the Fock intertwiners from those of the vector intertwiners.

\footnotetext{
${ }^{10}$ We have defined as $\Lambda^{(n)}=\varnothing$ for $n>h(\Lambda)$.
} 


\section{MacMahon $R$-matrix from the commutation of intertwiners}

Following the technique developed for the Fock representations, we can evaluate the commutator of two intertwining operators $\Xi_{\Pi}(K ; z)$, just constructed. For the moment, we omit the zero modes. The result is

$$
\begin{aligned}
\Xi_{\Pi}\left(K_{1} ; z_{1}\right) \Xi_{\Lambda}\left(K_{2} ; z_{2}\right)= & \Xi_{\Lambda}\left(K_{2} ; z_{2}\right) \Xi_{\Pi}\left(K_{1} ; z_{1}\right) \frac{\Upsilon\left(K_{1}, K_{2} \mid z_{1}, z_{2}\right)}{R_{\Pi \Lambda}^{K_{1}, K_{2}}\left(\frac{z_{1}}{z_{2}}\right)} \times \\
& \times K_{1}^{-\frac{|\Lambda|}{2}} K_{2}^{-\frac{|\Pi|}{2}} \prod_{(i, j, k) \in \Pi} \frac{\theta_{q_{3}}\left(K_{2} \frac{z_{2}}{z_{1}} q_{1}^{-i} q_{2}^{-j} q_{3}^{-k}\right)}{\theta_{q_{3}}\left(\frac{z_{2}}{z_{1}} q_{1}^{-i} q_{2}^{-j} q_{3}^{-k}\right)} \prod_{(a, b, c) \in \Lambda} \frac{\theta_{q_{3}}\left(\frac{z_{2}}{z_{1}} q_{1}^{a} q_{2}^{b} q_{3}^{c}\right)}{\theta_{q_{3}}\left(K_{1}^{-1} \frac{z_{2}}{z_{1}} q_{1}^{a} q_{2}^{b} q_{3}^{c}\right)},
\end{aligned}
$$

where

$$
\begin{array}{r}
\Upsilon_{q_{1}, q_{2}, q_{3}}\left(K_{1}, K_{2} \mid z_{1}, z_{2}\right)=\exp \left[\sum _ { n \geq 1 } \frac { 1 } { n } \frac { 1 } { ( 1 - q _ { 1 } ^ { n } ) ( 1 - q _ { 2 } ^ { n } ) ( 1 - q _ { 3 } ^ { n } ) ^ { 2 } } \left(\left(\frac{z_{2}}{z_{1}}\right)^{n}\left(1-K_{1}^{-n}\right)\left(1-K_{2}^{n}\right)-\right.\right. \\
\left.\left.-\left(\frac{z_{1}}{z_{2}}\right)^{n}\left(1-K_{1}^{n}\right)\left(1-K_{2}^{-n}\right)\right)\right], \quad(5.2)
\end{array}
$$

and $\theta_{q}(x)=\prod_{k>0}\left(1-q^{k+1}\right)\left(1-q^{k} x\right)\left(1-q^{k+1} x^{-1}\right)$. The extra theta-functions in eq. (5.1) are in fact precisely cancelled by the zero modes of the intertwiners. Notice that they depend on the states $\Pi$ or $\Lambda$ separately (though the dependence on the both spectral parameters is nontrivial).

We conclude that the commutation relations for the intertwiners that we have constructed in section 4 indeed feature the MacMahon $R$-matrix from section 3 . The whole picture of the intertwiners and $R$-matrices which we have presented in this paper is therefore consistent.

\section{Conclusions}

In this paper, we have introduced novel intertwining operators for the DIM algebra. These operators feature the MacMahon representations (representations on plane partitions), whose role in the DIM algebra is analogous to the role of free field representations for (quantum) affine Lie algebras. Our intertwining operators generalize the refined topological vertices [16-23]: they depend on a pair of ordinary Young diagrams and on one $3 d$ Young diagram, and on an extra parameter: the central charge associated with the MacMahon representation. For quantized values of the central charge, our vertices reproduce the refined topological vertices, or a "strip" combination thereof.

We also write down explicitly the DIM $R$-matrix acting on the tensor product of MacMahon representations (assuming their central charges are aligned along the preferred direction) and prove that it determines the commutation relations of the intertwiners.

The next logical step on our way is to build the generalization of the network formalism $[8,41]$ with some legs having the MacMahon representations and investigate the 
resulting partition functions and corresponding constraint algebras [8,41-44]. It is also very interesting to understand if there is a corresponding Type IIB string construction. Since a pair of central charges of the DIM representation corresponds to the $(p, q)$-charges of the 5 -brane in Type IIB, it is not immediately clear what would the MacMahon representation with an arbitrary (non-integer) central charge correspond to.

Using the technique of $[45,46]$, one can derive the $(q, t)-\mathrm{KZ}$ equations for the DIM intertwiners containing not only Fock representations, but the MacMahon ones too. However, in this approach, the MacMahon representations are restricted to lie only on the "vertical" legs. It would be interesting to lift this restriction, but, to this end, one needs an intertwiner of several MacMahon representations, which have not yet been described. In [13], we found that the DIM $R$-matrix $\mathcal{R}_{\lambda \mu}(x)$ for the Fock representations appears in the difference equation

$$
N_{\lambda \mu}\left(\frac{q}{t} x\right)=\left(\frac{q}{t}\right)^{\frac{1}{2}(|\lambda|+|\mu|)} \mathcal{R}_{\lambda \mu}(x)^{-1} N_{\lambda \mu}(x),
$$

satisfied by the Nekrasov factor $N_{\lambda \mu}(x)$;

$$
\begin{aligned}
N_{\lambda \mu}(x) & =\prod_{(i, j) \in \lambda}\left(1-x q^{\lambda_{i}-j} t^{i-\mu_{j}^{\mathrm{T}}+1}\right) \prod_{(i, j) \in \mu}\left(1-x q^{-\mu_{i}+j-1} t^{-\lambda_{j}^{\mathrm{T}}+i}\right) \\
& =\exp \left[-\sum_{n \geq 1} \frac{1-t^{n}}{n\left(1-q^{n}\right)} x^{n} \sum_{i, j}\left(q^{n\left(\lambda_{i}-\mu_{j}\right)}-1\right) t^{n(j-i)}\right] .
\end{aligned}
$$

It is an interesting problem to solve a generalization of (6.1) with $\mathcal{R}_{\lambda \mu}(x)$ replaced by the MacMahon $R$-matrix $R_{\Pi \Lambda}^{K_{1}, K_{2}}(x)$. The solution should be regarded as a generalized Nekrasov factor $N_{\Pi \Lambda}^{K_{1}, K_{2}}(x)$. It is natural to expect that the factor is related to a norm of the triple Macdonald functions [25].

Another interesting aim might be the generalization of our story to the case of MacMahon modules with nontrivial asymptotics, i.e. to $3 d$ Young diagrams with nontrivial "ends" along the coordinate axes $[10,11]$. We think one can use the same strategy by making a judicious choice of the spectral parameters of constituent Fock representations. Thus, the formulas should be quite similar to those presented above.

Finally, one can try to build the most general representation of the DIM algebra, in which the both central charges (not just one, as in the MacMahon case) are completely arbitrary. To our knowledge, such representations have never been studied, so it might lead to some unexpected surprises.

\section{Acknowledgments}

Our work is supported in part by Grants-in-Aid for Scientific Research (17K05275) (H.A.), (15H05738, 18K03274) (H.K.) and JSPS Bilateral Joint Projects (JSPS-RFBR collaboration) "Elliptic algebras, vertex operators and link invariants" from MEXT, Japan. It is also partly supported by the grant of the Foundation for the Advancement of Theoretical 
Physics "BASIS" (A.Mir. and A.Mor.), by RFBR grants 19-01-00680 (A.Mir.) and 1902-00815 (A.Mor. and Y.Z.), by joint grants 19-51-53014-GFEN-a (A.Mir. and A.Mor.), 19-51-50008-YaF-a (A.Mir. and Y.Z.), 18-51-05015-Arm-a (A.Mir., A.Mor. and Y.Z.), 1851-45010-IND-a (A.Mir. and A.Mor.). The work of Y.Z. was supported in part by INFN and by the ERC Starting Grant 637844-HBQFTNCER.

\section{A Conventions and useful functions}

The intriguing triality of the quantum toroidal algebra $U_{\mathfrak{q}, \mathfrak{d}}\left(\widehat{\widehat{\mathfrak{g}}}_{1}\right)$ becomes manifest, when we use the parameters $\left(q_{1}, q_{2}, q_{3}\right)$ with $q_{1} q_{2} q_{3}=1$. These parameters are also natural from the point of view of plane partitions used in the MacMahon representation. In this paper, we use the following convention: ${ }^{11}$

$$
q_{1}=\mathfrak{d} \mathfrak{q}^{-1}=q, \quad q_{2}=\mathfrak{d}^{-1} \mathfrak{q}^{-1}=t^{-1}, \quad q_{3}=\mathfrak{q}^{2}=t / q .
$$

The parameters $(q, t)$ can be identified with those appear in the Macdonald functions. The formulas for the vertex operator representation can be expressed neatly by introducing the following factor:

$$
k_{r}=\prod_{i=1}^{3}\left(q_{i}^{r / 2}-q_{i}^{-r / 2}\right)=\prod_{i=1}^{3}\left(q_{i}^{r}-1\right)=\sum_{i=1}^{3}\left(q_{i}^{r}-q_{i}^{-r}\right)
$$

The function

$$
G(z)=\frac{\left(1-q_{1}^{-1} z\right)\left(1-q_{2}^{-1} z\right)\left(1-q_{3}^{-1} z\right)}{\left(1-q_{1} z\right)\left(1-q_{2} z\right)\left(1-q_{3} z\right)}, \quad G(z) G\left(z^{-1}\right)=1 .
$$

derived from the structure function $g(z, w)=\left(z-q_{1} w\right)\left(z-q_{2} w\right)\left(z-q_{3} w\right)$ of $U_{\mathfrak{q}, \mathfrak{d}}\left(\widehat{\widehat{\mathfrak{g l}}}_{1}\right)$ appears in the formula of the MacMahon representation. Starting from the fundamental rational function

$$
\psi(z)=\mathfrak{q} \frac{1-q_{3}^{-1} z}{1-z}=\psi\left(q_{3} / z\right)^{-1},
$$

we can reconstruct the function $G(x)$ as follows:

$$
\tilde{\psi}(z)=\psi(z) \psi\left(q_{2}^{-1} z\right)^{-1}, \quad G(z)=\tilde{\psi}(z) \tilde{\psi}\left(q_{1}^{-1} z\right)^{-1} .
$$

These functions appear in the generating functions of the eigenvalues of $K^{ \pm}(z)$ in the vertical representations. On the other hand, in the vertex operator representation, the function $G(z)$ arise as

$$
G(z)=s\left(\mathfrak{q}^{-1} z\right) s(\mathfrak{q} z)^{-1}
$$

from the scattering factor

$$
s(z)=\frac{(1-\mathfrak{q} z)\left(1-\mathfrak{q}^{-1} z\right)}{(1-\mathfrak{d} z)\left(1-\mathfrak{d}^{-1} z\right)}
$$

in the OPE relation of the vertex operators.

\footnotetext{
${ }^{11}$ The parameters $q_{2}$ and $q_{3}$ are exchanged, compared with [26] and [11].
} 


\section{B Properties of $\theta$-function}

We naturally encounter the following $\theta$-function with parameter $p=q_{3}=\mathfrak{q}^{2}$ in the construction of the MacMahon intertwiner:

$$
\begin{aligned}
\theta_{p}(z) & :=(p ; p)_{\infty}(z ; p)_{\infty}\left(p z^{-1} ; p\right)_{\infty}=(1-z) \prod_{k=1}^{\infty}\left(1-p^{k}\right)\left(1-p^{k} z\right)\left(1-p^{k} z^{-1}\right) \\
(z ; p)_{\infty} & :=\prod_{k=0}^{\infty}\left(1-p^{k} z\right) .
\end{aligned}
$$

From the Jacobi triple product formula

$$
\theta_{p}(z)=\sum_{n \in \mathbb{Z}} p^{\frac{n}{2}(n-1)}(-z)^{n}
$$

we see $\theta_{p}(z)$ is an elliptic function with quasi double periodicity. In this paper, only ratios of $\theta$-functions appear in the formula, for example see (4.69), hence we can omit the factor $(p ; p)_{\infty}$ in (B.1) safely. The function $\theta_{p}(z)$ satisfies the following relations

$$
\begin{aligned}
& \theta_{p}\left(z^{-1}\right)=-z^{-1} \theta_{p}(z), \\
& \theta_{p}\left(z p^{n}\right)=(-z)^{-n} p^{-\frac{n}{2}(n-1)} \theta_{p}(z), \quad n \in \mathbb{Z} .
\end{aligned}
$$

\section{Zero modes of the Fock intertwiner}

In this section, we write down the zero modes of the Fock space intertwining operators [15] in a more convenient notation.

The horizontal Fock representations are characterized by the integer values of the two central charges: $K_{1}=q_{3}=\frac{t}{q}$ and $K_{2}=\frac{t}{q}^{N}$, and by the complex spectral parameter $u$. The zero modes of the Fock representation of the DIM algebra should therefore be written as operators acting on the vectors $|N, u\rangle$. Let us introduce an operator $P_{1}$, which by definition gives the eigenvalue $u$ when acting on $|N, u\rangle$ :

$$
P_{1}|N, u\rangle=u|N, u\rangle .
$$

Let us write the DIM generators $x^{ \pm}(z), \psi^{ \pm}(z)$ from [15] in terms of $P_{1}$, and $c_{2}=\log _{q_{3}} K_{2}$ :

$$
\begin{aligned}
& x^{+}(z)=P_{1}\left(\sqrt{\frac{q}{t} z}\right)^{-c_{2}} \eta(z), \\
& x^{-}(z)=P_{1}^{-1}\left(\sqrt{\frac{q}{t} z}\right)^{c_{2}} \xi(z), \\
& \psi^{+}(z)=\left(\frac{q}{t}\right)^{\frac{c_{2}}{2}} \varphi^{+}(z), \\
& \psi^{-}(z)=\left(\frac{q}{t}\right)^{-\frac{c_{2}}{2}} \varphi^{-}(z),
\end{aligned}
$$

where $\eta(z), \xi(z)$ and $\varphi^{ \pm}(z)$ denote the exponentials of the free boson (non-zero) modes. 
The intertwiner $\Psi(w)$ with the vertical Fock representation of central charges $K_{1}=1$, $K_{2}=\frac{t}{q}$ and the spectral parameter $w$ changes both the spectral parameter and the central charge of the horizontal representation. To account for this, we introduce the operators $Q_{1}$ and $e_{2}$ canonically conjugate to $P_{1}$, and $c_{2}$ :

$$
\begin{aligned}
Q_{1}|u, N\rangle & =u \frac{\partial}{\partial u}|u, N\rangle, \\
e_{2}|u, N\rangle & =|u, N+1\rangle .
\end{aligned}
$$

The intertwiner is then given by

$$
\Phi_{\lambda}(w)=e_{2}(-w)^{Q_{1}} \prod_{(i, j) \in \lambda}\left(\left(-w P_{1}\right)\left(w q^{j-\frac{1}{2}} t^{\frac{1}{2}-i}\right)^{-c_{2}-1}\right) \widetilde{\Phi}_{\lambda}(w)
$$

where $\widetilde{\Phi}_{\lambda}(w)$ contains only nonzero bosonic modes.

It is easy to derive the commutation relations of the intertwiners with the generators, as well as between the intertwiners themselves from the canonical commutation relations

$$
\begin{aligned}
Q_{1} P_{1} & =P_{1}\left(Q_{1}+1\right), \\
c_{2} e_{2} & =e_{2}\left(c_{2}+1\right) .
\end{aligned}
$$

Notice that only part of the zero modes in eqs. (C.8) depends on the diagram living in the vertical Fock module: the essential part (conjugate to $P_{1}$ and $c_{2}$ ) is independent of the diagram.

Open Access. This article is distributed under the terms of the Creative Commons Attribution License (CC-BY 4.0), which permits any use, distribution and reproduction in any medium, provided the original author(s) and source are credited.

\section{References}

[1] J.-t. Ding and K. Iohara, Generalization and deformation of Drinfeld quantum affine algebras, Lett. Math. Phys. 41 (1997) 181 [INSPIRE].

[2] K. Miki, $A(q, \gamma)$ analog of the $W_{1+\infty}$ algebra, J. Math. Phys. 48 (2007) 123520.

[3] V. Ginzburg, M. Kapranov and E. Vasserot, Langlands reciprocity for algebraic surfaces, Math. Res. Lett. 2 (1995) 147 [q-alg/9502013].

[4] M. Varagnolo and E. Vasserot, Schur duality in the toroidal setting, Commun. Math. Phys. 182 (1996) 469 [q-alg/9506026].

[5] R.V. Moody, S.E. Rao and T. Yokonuma, Toroidal Lie algebras and vertex representations, Geom. Dedicata 35 (1990) 283.

[6] G. Lockhart and C. Vafa, Superconformal Partition Functions and Non-perturbative Topological Strings, JHEP 10 (2018) 051 [arXiv: 1210.5909] [INSPIRE].

[7] A. Iqbal and K. Shabbir, Elliptic CY3folds and Non-Perturbative Modular Transformation, Eur. Phys. J. C 76 (2016) 148 [arXiv:1510.03332] [InSPIRE]. 
[8] A. Mironov, A. Morozov and Y. Zenkevich, Ding-Iohara-Miki symmetry of network matrix models, Phys. Lett. B 762 (2016) 196 [arXiv:1603.05467] [InSPIRE].

[9] F. Nieri, Y. Pan and M. Zabzine, q-Virasoro modular triple, Commun. Math. Phys. 366 (2019) 397 [arXiv:1710.07170] [INSPIRE].

[10] M. Bershtein, B. Feigin and G. Merzon, Plane partitions with a "pit": Generating functions and representation theory, Selecta Math. 24 (2018) 21 [arXiv:1512.08779].

[11] B. Feigin, M. Jimbo, T. Miwa and E. Mukhin, Quantum toroidal $\mathfrak{g l}_{1}$ algebra: plane partitions, Kyoto J. Math. 52 (2012) 621 [arXiv:1110.5310].

[12] H. Awata et al., Toric Calabi-Yau threefolds as quantum integrable systems. $\mathcal{R}$-matrix and $\mathcal{R} \mathcal{T} \mathcal{T}$ relations, JHEP 10 (2016) 047 [arXiv: 1608. 05351] [INSPIRE].

[13] H. Awata et al., Anomaly in $\mathcal{R} \mathcal{T} \mathcal{T}$ relation for DIM algebra and network matrix models, Nucl. Phys. B 918 (2017) 358 [arXiv:1611.07304] [INSPIRE].

[14] A. Smirnov, On the Instanton R-matrix, Commun. Math. Phys. 345 (2016) 703 [arXiv: 1302.0799] [INSPIRE].

[15] H. Awata, B. Feigin and J. Shiraishi, Quantum Algebraic Approach to Refined Topological Vertex, JHEP 03 (2012) 041 [arXiv:1112.6074] [INSPIRE].

[16] A. Iqbal, C. Kozcaz and C. Vafa, The Refined topological vertex, JHEP 10 (2009) 069 [hep-th/0701156] [INSPIRE].

[17] S. Gukov, A. Iqbal, C. Kozcaz and C. Vafa, Link Homologies and the Refined Topological Vertex, Commun. Math. Phys. 298 (2010) 757 [arXiv:0705.1368] [InSPIRE].

[18] $\mathrm{H}$. Awata and $\mathrm{H}$. Kanno, Instanton counting, Macdonald functions and the moduli space of D-branes, JHEP 05 (2005) 039 [hep-th/0502061] [INSPIRE].

[19] H. Awata and H. Kanno, Refined BPS state counting from Nekrasov's formula and Macdonald functions, Int. J. Mod. Phys. A 24 (2009) 2253 [arXiv:0805.0191] [InSPIRE].

[20] H. Awata and H. Kanno, Changing the preferred direction of the refined topological vertex, J. Geom. Phys. 64 (2013) 91 [arXiv:0903.5383] [INSPIRE].

[21] M. Taki, Refined Topological Vertex and Instanton Counting, JHEP 03 (2008) 048 [arXiv:0710.1776] [INSPIRE].

[22] M. Taki, Flop Invariance of Refined Topological Vertex and Link Homologies, arXiv:0805.0336 [INSPIRE].

[23] H. Awata and H. Kanno, Macdonald operators and homological invariants of the colored Hopf link, J. Phys. A 44 (2011) 375201 [arXiv:0910.0083] [INSPIRE].

[24] I.G. Macdonald, Symmetric functions and Hall polynomials, second edition, Oxford Mathematical Monographs, Oxford University Press, Oxford U.K. (1995).

[25] Y. Zenkevich, 3d field theory, plane partitions and triple Macdonald polynomials, arXiv: 1712.10300 [INSPIRE].

[26] B. Feigin, E. Feigin, M. Jimbo, T. Miwa and E. Mukhin, Quantum continuous $\mathfrak{g l}_{\infty}$ : Semi-infinite construction of representations, Kyoto J. Math. 51 (2011) 337 [arXiv: 1002.3100].

[27] B. Feigin, M. Jimbo, T. Miwa and E. Mukhin, Finite Type Modules and Bethe Ansatz for Quantum Toroidal $\mathfrak{g l}_{1}$, Commun. Math. Phys. 356 (2017) 285 [arXiv:1603.02765] [INSPIRE]. 
[28] B. Feigin, K. Hashizume, A. Hoshino, J. Shiraishi and S. Yanagida, A commutative algebra on degenerate $\mathbb{C P}^{1}$ and Macdonald polynomials, J. Math. Phys. 50 (2009) 095215 [arXiv:0904.2291].

[29] A. Tsymbaliuk, The affine Yangian of $\mathfrak{g l}_{1}$ revisited, Adv. Math. 304 (2017) 583 [arXiv: 1404.5240] [INSPIRE].

[30] E. Mukhin, V. Tarasov and A. Varchenko, Bispectral and $\left(\mathfrak{g l}_{N}, \mathfrak{g l}_{M}\right)$ Dualities, math.QA/0510364.

[31] E. Mukhin, V. Tarasov and A. Varchenko, Bispectral and $\left(\mathfrak{g l}_{N}, \mathfrak{g l}_{M}\right)$ dualities, discrete versus differential, Adv. Math. 218 (2008) 216 [math.QA/0605172].

[32] A. Mironov, A. Morozov, Y. Zenkevich and A. Zotov, Spectral Duality in Integrable Systems from AGT Conjecture, JETP Lett. 97 (2013) 45 [arXiv: 1204.0913] [INSPIRE].

[33] A. Mironov, A. Morozov, B. Runov, Y. Zenkevich and A. Zotov, Spectral Duality Between Heisenberg Chain and Gaudin Model, Lett. Math. Phys. 103 (2013) 299 [arXiv:1206.6349] [INSPIRE].

[34] A. Mironov, A. Morozov, B. Runov, Y. Zenkevich and A. Zotov, Spectral dualities in XXZ spin chains and five dimensional gauge theories, JHEP 12 (2013) 034 [arXiv:1307.1502] [INSPIRE].

[35] L. Bao, E. Pomoni, M. Taki and F. Yagi, M5-Branes, Toric Diagrams and Gauge Theory Duality, JHEP 04 (2012) 105 [arXiv: 1112.5228] [INSPIRE].

[36] M. Aganagic, N. Haouzi, C. Kozcaz and S. Shakirov, Gauge/Liouville Triality, arXiv: 1309.1687 [INSPIRE].

[37] M. Aganagic, N. Haouzi and S. Shakirov, $A_{n}$-Triality, arXiv:1403.3657 [INSPIRE].

[38] M. Aganagic and N. Haouzi, ADE Little String Theory on a Riemann Surface (and Triality), arXiv: 1506.04183 [INSPIRE].

[39] T. Procházka, $\mathcal{W}$-symmetry, topological vertex and affine Yangian, JHEP 10 (2016) 077 [arXiv: 1512.07178] [INSPIRE].

[40] J.-E. Bourgine, M. Fukuda, K. Harada, Y. Matsuo and R.-D. Zhu, $(p, q)$-webs of DIM representations, $5 d \mathcal{N}=1$ instanton partition functions and qq-characters, JHEP 11 (2017) 034 [arXiv: 1703.10759] [INSPIRE].

[41] H. Awata et al., Explicit examples of DIM constraints for network matrix models, JHEP 07 (2016) 103 [arXiv : 1604.08366] [INSPIRE].

[42] T. Kimura and V. Pestun, Quiver W-algebras, Lett. Math. Phys. 108 (2018) 1351 [arXiv: 1512.08533] [INSPIRE].

[43] J.-E. Bourgine, M. Fukuda, Y. Matsuo, H. Zhang and R.-D. Zhu, Coherent states in quantum $\mathcal{W}_{1+\infty}$ algebra and qq-character for 5d Super Yang-Mills, Prog. Theor. Exp. Phys. 2016 (2016) 123B05 [arXiv: 1606.08020] [INSPIRE].

[44] A. Nedelin and M. Zabzine, q-Virasoro constraints in matrix models, JHEP 03 (2017) 098 [arXiv: 1511.03471] [INSPIRE].

[45] H. Awata et al., Generalized Knizhnik-Zamolodchikov equation for Ding-Iohara-Miki algebra, Phys. Rev. D 96 (2017) 026021 [arXiv: 1703.06084] [InSPIRE].

[46] H. Awata, H. Kanno, A. Mironov, A. Morozov, K. Suetake and Y. Zenkevich, $(q, t)-K Z$ equations for quantum toroidal algebra and Nekrasov partition functions on ALE spaces, JHEP 03 (2018) 192 [arXiv:1712.08016] [INSPIRE]. 